\title{
Approximate Controllability of Fractional Delay Dynamic Inclusions with Nonlocal Control Conditions*
}

\author{
Amar Debbouche $e^{a, b}$ \\ amar_debbouche@yahoo.fr \\ Delfim F. M. Torres ${ }^{b}$ \\ delfim@ua.pt \\ ${ }^{a}$ Department of Mathematics, Guelma University, Guelma, Algeria \\ ${ }^{b}$ CIDMA-Center for Research and Development in Mathematics and Applications, \\ Department of Mathematics, University of Aveiro, 3810-193 Aveiro, Portugal
}

\begin{abstract}
We introduce a nonlocal control condition and the notion of approximate controllability for fractional order quasilinear control inclusions. Approximate controllability of a fractional control nonlocal delay quasilinear functional differential inclusion in a Hilbert space is studied. The results are obtained by using the fractional power of operators, multi-valued analysis, and Sadovskii's fixed point theorem. Main result gives an appropriate set of sufficient conditions for the considered system to be approximately controllable. As an example, a fractional partial nonlocal control functional differential inclusion is considered.
\end{abstract}

2010 Mathematics Subject Classification: 26A33; 34A60; 34G25; 93B05.

Keywords: approximate controllability; control theory; multivalued maps; fractional dynamic inclusions; fractional power; fixed points; semigroup theory.

\section{Introduction}

We are concerned with the fractional delay quasilinear control inclusion

$$
D_{t}^{\alpha}[u(t)-g(t, u(\sigma(t)))] \in A u(t)+\int_{0}^{t} f\left(t, s, B_{1} \mu_{1}(\delta(s))\right) d s
$$

subject to the nonlocal control condition

$$
u(0)+h(u(t))=B_{2} \mu_{2}(t)+u_{0},
$$

where the unknown $u(\cdot)$ takes its values in a Hilbert space $H$ with norm $\|\cdot\|, D_{t}^{\alpha}$ is the Caputo fractional derivative with $0<\alpha \leq 1$ and $t \in J=[0, a]$. Let $A$ be a closed linear operator defined on a dense domain $D(A)$ in $H$ into $H$ that generates an analytic semigroup $Q(t), t \geq 0$, of bounded linear operators on $H$ and $u_{0} \in D(A)$. We assume that $\left\{B_{i}: U \rightarrow H, i=1,2\right\}$ is a family of bounded linear operators, the control functions $\mu_{i}, i=1,2$, belong to the space $L^{2}(J, U)$, a Hilbert space of admissible control functions with $U$ as Hilbert space, and $\sigma, \delta: J \rightarrow J^{\prime}$ are delay arguments, $J^{\prime}=[0, t]$. It is also assumed that $g: J \times H \rightarrow H$ and $h: C\left(J^{\prime}: H\right) \rightarrow H$ are given abstract functions and $f: \Delta \times H \rightarrow H$ is a multi-valued map, $\Delta=\{(t, s): 0 \leq s \leq t \leq a\}$.

Three centuries ago, fractional calculus (i.e., the calculus of non-integer order derivatives and integrals) has been dealt almost by mathematicians only. During the past decades, this subject

${ }^{*}$ This is a preprint of a paper whose final and definite form will be published in Applied Mathematics and Computation, ISSN 0096-3003 (see http://www.sciencedirect.com/science/journal/00963003). Submitted 12/Feb/2013; Revised 04/May/2014; Accepted 25/May/2014. 
and its potential applications have gained a lot of importance, mainly because fractional calculus has become a powerful tool with more accurate and successful results in modeling several complex phenomena in numerous seemingly diverse and widespread fields of science and engineering [5. $[10,13,18,22,31,33,34$. It was found that various, especially interdisciplinary applications, can be elegantly modeled with the help of fractional derivatives. Several authors have demonstrated applications in the frequency dependent damping behavior of viscoelastic materials [3]4, dynamics of interfaces between nanoparticles and substrates [9, the nonlinear oscillation of earthquakes [21, bioengineering [29, continuum and statistical mechanics [30, signal processing [36, filter design, robotics and circuit theory [42. Fractional differential equations provide an excellent instrument for the description of memory and hereditary properties of various materials and processes [39.

In control theory, one of the most important qualitative aspects of a dynamical control system is controllability. The problem of controllability consists to show the existence of a control function that steers the solution of the system from its initial state to a final state, where the initial and final states may vary over the entire space. A large class of scientific and engineering problems is modeled by partial differential equations, integral equations or coupled ordinary and partial differential, integrodifferential equations, which arise in problems connected with heat-flow in materials with memory, viscoelasticity and many other physical phenomena. So it becomes important to study controllability results of such systems using available techniques. The concept of controllability plays a major role in finite-dimensional control theory, so that it is natural to try to generalize it to infinite dimensions [40. Moreover, the exact controllability for semilinear fractional order systems, when the nonlinear term is independent of the control function, is proved by assuming that the controllability operator has an induced inverse on a quotient space, see for example [11,12]. However, if the semigroup associated with the system is compact, then the controllability operator is also compact and hence the induced inverse does not exist because the state space is infinite dimensional [50]. Thus, the concept of exact controllability is too strong and has limited applicability, while approximate controllability is a weaker concept completely adequate in applications.

In recent years, attention has been paid to establish sufficient conditions for the existence and controllability of (fractional) differential equations and inclusions, see, for instance, $1,2,2,7,8,28$, 46, 51. Ntouyas and O'Regan [35] studied existence results for semilinear neutral functional differential inclusions, $\mathrm{Fu}[20$ established approximate controllability for neutral nonlocal impulsive differential inclusions and Yan [52,53 investigated the question of approximate controllability of both fractional neutral functional differential equations and fractional integro-differential inclusions with state-dependent delays. For more works about the approximate controllability for fractional systems, we refer the reader to [15,41,43, 45, 47, 48, see also Kumar and Sukavanam 26, 49. However, in the mentioned papers, the control function is located only in the inhomogeneous part of the evolution system. For this reason, and motivated by this fact, we construct here two control functions: the first control depends on the multi-valued map and the other with the nonlocal condition. In order to realize this new complex form, we introduce here the study of approximate controllability for a class of fractional delay dynamic inclusions with nonlocal control conditions.

The paper is organized as follows. In Section 2, we review some essential facts from fractional calculus, multi-valued analysis and semigroup theory, that are used to obtain our main results. In Section 3, we state and prove existence and approximate controllability results for the fractional control system (11)-(2). Finally, in Section 4, as an example, a fractional partial dynamical differential inclusion with a nonlocal control condition is considered. We end with Section 5 of conclusions and some possible future directions of research.

\section{Preliminaries}

In this section we give some basic definitions, notations, propositions and lemmas, which will be used throughout the work. In particular, we state main properties of fractional calculus [25,32,39], elementary principles of multi-valued analysis [16, 24, and well known facts in semigroup theory [23, 38, 54. 
Definition 1. The fractional integral of order $\alpha>0$ of a function $f \in L^{1}\left([a, b], \mathbb{R}^{+}\right)$is given by

$$
I_{a}^{\alpha} f(t)=\frac{1}{\Gamma(\alpha)} \int_{a}^{t}(t-s)^{\alpha-1} f(s) d s,
$$

where $\Gamma$ is the gamma function. If $a=0$, we can write $I^{\alpha} f(t):=I_{0}^{\alpha} f(t)=\left(g_{\alpha} * f\right)(t)$, where

$$
g_{\alpha}(t):= \begin{cases}\frac{1}{\Gamma(\alpha)} t^{\alpha-1}, & t>0 \\ 0, & t \leq 0\end{cases}
$$

and, as usual, $*$ denotes the convolution of functions.

Remark 2. For function (3), one has $\lim _{\alpha \rightarrow 0} g_{\alpha}(t)=\delta(t)$ with $\delta$ the delta Dirac function.

Definition 3. The Riemann-Liouville fractional derivative of order $n-1<\alpha<n, n \in \mathbb{N}$, for a function $f \in C([0, \infty))$ is given by

$$
{ }^{L} D^{\alpha} f(t)=\frac{1}{\Gamma(n-\alpha)} \frac{d^{n}}{d t^{n}} \int_{0}^{t} \frac{f(s)}{(t-s)^{\alpha+1-n}} d s, \quad t>0 .
$$

Definition 4. The Caputo fractional derivative of order $n-1<\alpha<n, n \in \mathbb{N}$, for a function $f \in C^{n-1}([0, \infty))$ is given by

$$
{ }^{C} D^{\alpha} f(t)={ }^{L} D^{\alpha}\left(f(t)-\sum_{k=0}^{n-1} \frac{t^{k}}{k !} f^{(k)}(0)\right), \quad t>0 .
$$

Remark 5. The following properties hold (see, e.g., [39]):

(i) If $f \in C^{n}([0, \infty))$, then

$$
{ }^{C} D^{\alpha} f(t)=\frac{1}{\Gamma(n-\alpha)} \int_{0}^{t} \frac{f^{(n)}(s)}{(t-s)^{\alpha+1-n}} d s=I^{n-\alpha} f^{n}(t), \quad t>0, \quad n-1<\alpha<n, \quad n \in \mathbb{N} .
$$

(ii) The Caputo derivative of a constant is equal to zero.

(iii) If $f$ is an abstract function with values in $H$, then the integrals that appear in Definitions 14 4 are taken in Bochner's sense.

According to previous definitions, it is suitable to rewrite problem (11)-(2) as the equivalent integral inclusion

$$
\begin{aligned}
u(t) \in B_{2} \mu_{2}(t)+u_{0}-h(u(t)) & -g(0, u(\sigma(0)))+g\left(t, u\left(\sigma_{1}(t)\right)\right) \\
& +\frac{1}{\Gamma(\alpha)} \int_{0}^{t}(t-s)^{\alpha-1}\left[A u(s)+\int_{0}^{s} f\left(s, \eta, B_{1} \mu_{1}(\delta(\eta))\right) d \eta\right] d s,
\end{aligned}
$$

provided the integral in (4) exists. Before formulating the definition of mild solution to (11)-(2), we first give the following notations, corollaries and lemmas.

Let $(X,\|\cdot\|)$ be a Banach space, $C(J, X)$ denote the Banach space of continuous functions from $J$ into $X$ with the norm $\|u\|_{J}=\sup \{\|u(t)\|: t \in J\}$, and let $\mathcal{L}(X)$ be the Banach space of bounded linear operators from $X$ to $X$ with the norm $\|G\|_{\mathcal{L}(X)}=\sup \{\|G(u)\|:\|u\|=1\}$. We also denote:

- $P(X)=\left\{Y \in 2^{X}: Y \neq \emptyset\right\}$,

- $P_{c l} X=\{Y \in P(X), Y$ is closed $\}$,

- $P_{b} X=\{Y \in P(X), Y$ is bounded $\}$, 
- $P_{c} X=\{Y \in P(X), Y$ is convex $\}$,

- $P_{c p} X=\{Y \in P(X), Y$ is compact $\}$.

The following results on multi-valued analysis are useful.

Proposition 6 (See [16]). (1) A measurable function $u: J \rightarrow X$ is Bochner integrable if and only if $\|u\|$ is Lebesgue integrable.

(2) A multi-valued map $F: X \rightarrow 2^{X}$ is said to be convex-valued (closed-valued) if $F(u)$ is convex (closed) for all $u \in X$; is said to be bounded on bounded sets if $F(B)=\bigcup_{u \in B} F(u)$ is bounded in $X$ for all $B \in P_{b}(X)$.

(3) A map $F$ is said to be upper semi-continuous (u.s.c.) on $X$ if for each $u_{0} \in X$ the set $F\left(u_{0}\right)$ is a nonempty closed subset of $X$, and if for each open subset $\Omega$ of $X$ containing $F\left(u_{0}\right)$, there exists an open neighborhood $\nabla$ of $u_{0}$ such that $F(\nabla) \subseteq \Omega$.

(4) $A$ map $F$ is said to be completely continuous if $F(B)$ is relatively compact for every $B \in$ $P_{b}(X)$. If the multi-valued map $F$ is completely continuous with nonempty compact values, then $F$ is u.s.c. if and only if $F$ has a closed graph, i.e., $u_{n} \rightarrow u, y_{n} \rightarrow y, y_{n} \in F\left(u_{n}\right)$ imply $y \in F(u)$. We say that $F$ has a fixed point if there is $u \in X$ such that $u \in F(u)$.

(5) A multi-valued map $F: J \rightarrow P_{c l}(X)$ is said to be measurable if for each $u \in X$ the function $y: J \rightarrow \mathbb{R}$ defined by $y(t)=d(u, F(t))=\inf \{\|u-z\|, z \in F(t)\}$ is measurable.

(6) A multi-valued map $F: X \rightarrow 2^{X}$ is said to be condensing if for any bounded subset $B \subset X$ with $\beta(B) \neq 0$ we have $\beta(F(B))<\beta(B)$, where $\beta(\cdot)$ denotes the Kuratowski measure of non-compactness defined as follows: $\beta(B):=\inf \{d>0: B$ can be covered by a finite number of balls of radius $d\}$.

The following results are motivated by 27 .

Proposition 7 (Cf. 27]). Let $X$ be a Banach space and let $f: \Delta \times X \rightarrow P_{b, c l, c}(X)$ satisfy the following conditions:

1. for each $\mu_{1} \in L^{2}\left(J^{\prime}, U\right),\left(t, s, B_{1} \mu_{1}\right) \rightarrow f\left(t, s, B_{1} \mu_{1}\right)$ is measurable on $\Delta$;

2. for each $(t, s) \in \Delta,\left(t, s, B_{1} \mu_{1}\right) \rightarrow f\left(t, s, B_{1} \mu_{1}\right)$ is u.s.c. with respect to $B_{1} \mu_{1}$;

3. for each fixed $\mu_{1} \in L^{2}\left(J^{\prime}, U\right)$, the set

$$
S_{f, \mu_{1}}=\left\{v \in L^{1}\left(J^{\prime}, X\right): B_{1} \mu_{1}(t)+v(\delta(t)) \in \int_{0}^{t} f\left(t, s, B_{1} \mu_{1}(\delta(s))\right) d s, \text { a.e. } t \in J\right\}
$$

is nonempty.

Also, let $P$ be a linear continuous mapping from $L^{1}\left(J^{\prime}, X\right)$ to $L^{2}\left(J^{\prime}, U\right)$. Then the operator

$$
\begin{aligned}
P \circ S_{f, \mu_{1}}: L^{2}\left(J^{\prime}, U\right) & \longrightarrow P_{c p, c}\left(L^{2}\left(J^{\prime}, U\right)\right) \\
\mu_{1} & \longmapsto P \circ S_{f}\left(\mu_{1}\right)=: P\left(S_{f, \mu_{1}}\right)
\end{aligned}
$$

is a closed graph operator.

Lemma 8 (See [16]). Let $\Omega$ be a bounded, convex, and closed set in the Banach space $X$ and $F: \Omega \rightarrow 2^{\Omega} \backslash\{\emptyset\}$ be a u.s.c. condensing multi-valued map. If for every $u \in \Omega, F(u)$ is a closed and convex set in $\Omega$, then $F$ has a fixed point in $\Omega$. 
Throughout the paper, $(H,\|\cdot\|)$ is a separable Hilbert space. If $A: D(A) \subset H \rightarrow H$ is the infinitesimal generator of a compact analytic semigroup of uniformly bounded linear operators $Q(\cdot)$, then there exists a constant $M \geq 1$ such that $\|Q(t)\| \leq M$ for $t \geq 0$. Without loss of generality, we assume that $0 \in \rho(A)$, the resolvent set $A$. Then it is possible to define the fractional power $A^{q}$, for $0<q \leq 1$, as a closed linear operator on its domain $D\left(A^{q}\right)$ with inverse $A^{-q}$. Furthermore, the subspace $D\left(A^{q}\right)$ is dense in $H$ and the expression $\|u\|_{q}=\left\|A^{q} u\right\|, u \in D\left(A^{q}\right)$ defines a norm on $D\left(A^{q}\right)$. Hereafter, we denote by $H_{q}$ the Banach space $D\left(A^{q}\right)$ normed with $\|u\|_{q}$.

Lemma 9 (See [38). Let $A$ be the infinitesimal generator of an analytic semigroup $Q(t)$. If $0 \in \rho(A)$, then

(a) $Q(t): H \rightarrow D\left(A^{q}\right)$ for every $t>0$ and $q \geq 0$;

(b) $Q(t) A^{q} u=A^{q} Q(t) u$ for every $u \in D\left(A^{q}\right)$;

(c) the operator $A^{q} Q(t)$ is bounded and $\left\|A^{q} Q(t)\right\| \leq M_{q} t^{-q} e^{-\omega t}$ for every $t>0$;

(d) if $0<q \leq 1$ and $u \in D\left(A^{q}\right)$, then $\|Q(t) u-u\| \leq C_{q} t^{q}\left\|A^{q} u\right\|$.

Remark 10. We note that:

(i) $D\left(A^{q}\right)$ is a Hilbert space with the norm $\|u\|_{q}=\left\|A^{q} u\right\|$ for $u \in D\left(A^{q}\right)$.

(ii) If $0<p \leq q \leq 1$, then $D\left(A^{q}\right) \hookrightarrow D\left(A^{p}\right)$.

(iii) $A^{-q}$ is a bounded linear operator in $H$ with $D\left(A^{q}\right)=\operatorname{Im}\left(A^{-q}\right)$.

Let us consider the set $\Omega=\left\{u: u \in C\left(J, H_{q}\right), q \in(0,1)\right\}$, which is a Banach space with the norm $\|u\|_{\Omega}=\sup _{t \in J}\|u(t)\|_{q}$.

Definition 11 (Cf. [14,17 and [20,35,52,55]). A state function $u(t) \in \Omega$ is called a mild solution of (11)-(2) if $u(0)=B_{2} \mu_{2}(t)+u_{0}-h(u(t))$, the function $(t-s)^{\alpha-1} A T_{\alpha}(t-s) g(s, u(\sigma(s))), s \in J$, is integrable on $[0, t)$ for every $t \in J$, and for each control $\mu_{1} \in L^{2}(J, U)$ there exists a function $v \in L^{1}\left(J^{\prime}, H\right)$ such that $v(\delta(t))+B_{1} \mu_{1}(t) \in \int_{0}^{t} f\left(t, s, B_{1} \mu_{1}(\delta(s))\right) d s$ a.e. on $J$ and the following integral equation is satisfied:

$$
\begin{aligned}
& u(t)=S_{\alpha}(t) {\left[B_{2} \mu_{2}(t)+u_{0}-h(u(t))-g(0, u(\sigma(0)))\right]+g(t, u(\sigma(t))) } \\
&+\int_{0}^{t}(t-s)^{\alpha-1}\left\{A T_{\alpha}(t-s) g(s, u(\sigma(s)))+T_{\alpha}(t-s)\left[v(\delta(s))+B_{1} \mu_{1}(s)\right]\right\} d s,
\end{aligned}
$$

where

$$
\begin{gathered}
S_{\alpha}(t)=\int_{0}^{\infty} \zeta_{\alpha}(\theta) Q\left(t^{\alpha} \theta\right) d \theta, \quad T_{\alpha}(t)=\alpha \int_{0}^{\infty} \theta \zeta_{\alpha}(\theta) Q\left(t^{\alpha} \theta\right) d \theta, \\
\zeta_{\alpha}(\theta)=\frac{1}{\alpha} \theta^{-1-\frac{1}{\alpha}} \varpi_{\alpha}\left(\theta^{-\frac{1}{\alpha}}\right) \geq 0, \quad \varpi_{\alpha}(\theta)=\frac{1}{\pi} \sum_{n=1}^{\infty}(-1)^{n-1} \theta^{-\alpha n-1} \frac{\Gamma(n \alpha+1)}{n !} \sin (n \pi \alpha), \theta \in(0, \infty),
\end{gathered}
$$

with $\zeta_{\alpha}$ the probability density function defined on $(0, \infty)$, that is, $\zeta_{\alpha}(\theta) \geq 0, \theta \in(0, \infty)$, and $\int_{0}^{\infty} \zeta_{\alpha}(\theta) d \theta=1$.

Lemma 12 (See $[52,55])$. The operators $S_{\alpha}(t)$ and $T_{\alpha}(t)$ satisfy the following properties.

(a) For any fixed $t \geq 0, S_{\alpha}(t)$ and $T_{\alpha}(t)$ are linear and bounded operators, i.e., for any $u \in H$, $\left\|S_{\alpha}(t) u\right\| \leq M\|u\|$ and $\left\|T_{\alpha}(t) u\right\| \leq \frac{M \alpha}{\Gamma(1+\alpha)}\|u\|$.

(b) $\left\{S_{\alpha}(t), t \geq 0\right\}$ and $\left\{T_{\alpha}(t), t \geq 0\right\}$ are strongly continuous, i.e., for $u \in H$ and $0 \leq t_{1}<t_{2} \leq a$, we have $\left\|S_{\alpha}\left(t_{2}\right) u-S_{\alpha}\left(t_{1}\right) u\right\| \rightarrow 0$ and $\left\|T_{\alpha}\left(t_{2}\right) u-T_{\alpha}\left(t_{1}\right) u\right\| \rightarrow 0$ as $t_{1} \rightarrow t_{2}$.

(c) For every $t>0, S_{\alpha}(t)$ and $T_{\alpha}(t)$ are compact operators. 
(d) For any $u \in H, p \in(0,1)$ and $q \in(0,1), A T_{\alpha}(t) u=A^{1-p} T_{\alpha}(t) A^{p} u, t \in J$, and

$$
\left\|A^{q} T_{\alpha}(t)\right\| \leq \frac{\alpha M_{q} \Gamma(2-q)}{\Gamma(1+\alpha(1-q))} t^{-q \alpha}, \quad 0<t \leq a .
$$

Motivated by the recent works [20,26, 41, 47, 49,52, we make use of the following notions. Let $u_{a}\left(u(0) ; \mu_{1}, \mu_{2}\right)$ be the state value of (1)-(2) at terminal time $a$, corresponding to the controls $\mu_{1}$ and $\mu_{2}$ and the nonlocal control value $u(0)$. For every $u_{0} \in H$, we introduce the set

$$
\mathfrak{R}(a, u(0))=\left\{u_{a}\left(B_{1} \mu_{1}(t)+u_{0}-h(u(t)) ; \mu_{1}, \mu_{2}\right)(0): \mu_{1}(\cdot), \mu_{2}(\cdot) \in L^{2}(J, U)\right\},
$$

which is called the reachable set of system (11)-(2) at terminal time $a$. Its closure in $H$ is denoted by $\overline{\mathfrak{R}(a, u(0))}$.

Definition 13. The system (11)-(2) is said to be approximately controllable on $J$ if $\overline{\Re(a, u(0))}=H$, that is, given an arbitrary $\epsilon>0$, it is possible to steer from the point $u(0)$ at time $a$ all points in the state space $H$ within a distance $\epsilon$.

Consider the following linear nonlocal control fractional system:

$$
\begin{gathered}
D_{t}^{\alpha} u(t)=A u(t)+B_{1} \mu_{1}(t), \\
u(0)=u_{0}+B_{2} \mu_{2} .
\end{gathered}
$$

The approximate controllability for the linear nonlocal control fractional system (6)-(7) is a natural generalization of the notion of approximate controllability of a linear first-order control system $\left(\alpha=1\right.$ and $\left.B_{2}=0\right)$. It is convenient at this point to introduce the controllability operators associated with (6) -(7) as

$$
\begin{aligned}
& \Gamma_{0,1}^{a}=\int_{0}^{a}(a-s)^{\alpha-1} T_{\alpha}(a-s) B_{1} B_{1}^{*} T_{\alpha}^{*}(a-s) d s, \\
& \Gamma_{0,2}^{a}=S_{\alpha}(a) B_{2} B_{2}^{*} S_{\alpha}^{*}(a),
\end{aligned}
$$

where $S_{\alpha}^{*}(t), T_{\alpha}^{*}(t)$ and $B_{j}^{*}, j=1,2$, denote the adjoints of $S_{\alpha}(t), T_{\alpha}(t)$ and $B_{j}$, respectively. Moreover, we give the relevant operators

$$
\mathcal{R}\left(\lambda, \Gamma_{0, i}^{a}\right)=\left(\lambda I+\Gamma_{0, i}^{a}\right)^{-1}
$$

for $i=1,2$ and $\lambda>0$. It is straightforward to see that $\Gamma_{0,1}^{a}$ and $\Gamma_{0,2}^{a}$ are linear bounded operators.

Lemma 14 (See [6,52]). The fractional linear control system (6) -(7) is approximately controllable on $J$ if and only if $\lambda \mathcal{R}\left(\lambda, \Gamma_{0, i}^{a}\right) \rightarrow 0$ as $\lambda \rightarrow 0^{+}, i=1,2$, in the strong operator topology.

\section{Main Results}

We obtain existence and approximate controllability results for the fractional nonlocal control inclusion (11)-(2). We consider the following hypotheses:

$\left(\mathrm{H}_{1}\right)$ There exists a constant $p \in(0,1)$ such that the function $g(\cdot, \cdot)$ maps $[0, a] \times H_{q}$ into $H_{p+q}$ and $A^{p} g:[0, a] \times H_{q} \rightarrow H_{q}$ satisfies a Lipschitz condition, that is, there exists a constant $L_{1}>0$ such that

$$
\left\|A^{p} g\left(t_{1}, u_{1}\right)-A^{p} g\left(t_{2}, u_{2}\right)\right\|_{q} \leq L_{1}\left(\left|t_{1}-t_{2}\right|+\left\|u_{1}-u_{2}\right\|_{q}\right)
$$

for any $0 \leq t_{1}, t_{2} \leq a, u_{1}, u_{2} \in H_{q}$. Moreover, there exists a constant $L_{2}>0$ such that the inequality

$$
\left\|A^{p} g(t, u)\right\|_{q} \leq L_{2}
$$

holds for any $u \in H_{q}$. 
$\left(\mathrm{H}_{2}\right)$ The multi-valued map $f: \Delta \times H_{q} \rightarrow P_{c, c p}(H)$ satisfies the following conditions:

(i) function $f(t, s, \cdot): H_{q} \rightarrow P_{c, c p}(H)$ is u.s.c. for each $(t, s) \in \Delta$, function $f\left(\cdot, \cdot, B_{1} \mu_{1}\right)$ is measurable for each $\mu_{1} \in L^{2}\left(J^{\prime}, U_{q}\right)$, and the set

$$
S_{f, \mu_{1}}=\left\{v \in L^{1}\left(J^{\prime}, H\right): B_{1} \mu_{1}(t)+v(\delta(t)) \in \int_{0}^{t} f\left(t, s, B_{1} \mu_{1}(\delta(s))\right) d s \text { a.e. on } H_{q}\right\}
$$

is nonempty;

(ii) there exists a positive constant $\omega$ such that $\left\|\int_{0}^{t} f\left(t, s, B_{1} \mu_{1}(\delta(s))\right) d s\right\| \leq \omega$ for all $(t, s, \cdot) \in \Delta \times H_{q}$, where

$$
\left\|\int_{0}^{t} f\left(t, s, B_{1} \mu_{1}(\delta(s))\right) d s\right\|=\sup \left\{\|v\|: B_{1} \mu_{1}+v \in \int_{0}^{t} f\left(t, s, B_{1} \mu_{1}(\delta(s))\right) d s\right\} .
$$

$\left(\mathrm{H}_{3}\right)$ Function $h: C(J, H) \rightarrow H_{q}$ is a completely continuous map and there exists a positive constant $k$ such that $\|h(u)\|_{q} \leq k$.

$\left(\mathrm{H}_{4}\right)$ The delay arguments $\sigma, \delta: J \rightarrow J^{\prime}$ are absolutely continuous and satisfy $|\sigma(t)| \leq t,|\delta(t)| \leq t$, for every $t \in J$.

For $\lambda>0, u \in \Omega$, we define the operator $F^{\lambda}: \Omega \rightarrow 2^{\Omega}$ as follows:

$$
\begin{aligned}
F^{\lambda}(u) & =\left\{z \in \Omega: z(t)=S_{\alpha}(t)\left[B_{2} \mu_{2}(t)+u_{0}-h(u(t))-g(0, u(\sigma(0)))\right]+g(t, u(\sigma(t)))\right. \\
& \left.+\int_{0}^{t}(t-s)^{\alpha-1}\left\{A T_{\alpha}(t-s) g(s, u(\sigma(s)))+T_{\alpha}(t-s)\left[v(\delta(s))+B_{1} \mu_{1}(s)\right]\right\} d s, v \in S_{f, \mu_{1}}\right\} .
\end{aligned}
$$

For any $u(\cdot) \in \Omega, u_{a} \in H$, we take the controls

$$
\mu_{1}=B_{1}^{*} T_{\alpha}^{*}(a-t) \mathcal{R}\left(\lambda, \Gamma_{0,1}^{a}\right) P(u(\cdot)), \quad \mu_{2}=B_{2}^{*} S_{\alpha}^{*}(a) \mathcal{R}\left(\lambda, \Gamma_{0,2}^{a}\right) P(u(\cdot)),
$$

where

$$
\begin{aligned}
P(u(\cdot))=u_{a}-S_{\alpha}(a) & {\left[u_{0}-h(u(t))-g(0, u(\sigma(0)))\right]-g(t, u(\sigma(t))) } \\
& -\int_{0}^{a}(a-s)^{\alpha-1}\left\{A T_{\alpha}(a-s) g(s, u(\sigma(s)))+T_{\alpha}(a-s) v(\delta(s))\right\} d s .
\end{aligned}
$$

We note that the fixed points of $F^{\lambda}$ are mild solutions of the fractional nonlocal control inclusion (1)-(2) .

Theorem 15. Let $u_{0} \in H_{q}$. If hypotheses $\left(H_{1}\right)-\left(H_{4}\right)$ are satisfied, then $F^{\lambda}$ has a fixed point on $J$ for each $\lambda>0$, provided $L_{1}\left[M\left\|A^{-p}\right\|+\left\|A^{-p}\right\|+\frac{a^{p \alpha}}{p \alpha} \frac{\alpha M_{1-p} \Gamma(1+p)}{\Gamma(1+\alpha p)}\right]<1$.

Proof. In order to prove the existence of mild solutions for system (11)-(2), we divide the proof into several steps.

Step 1. For each $0<\lambda<1, F^{\lambda}(u)$ is bounded. Using (12), Lemma 12, (8) and (9), we get

$$
\left\|\mu_{1}\right\| \leq \frac{1}{\lambda} \frac{M}{\Gamma(\alpha)}\left\|B_{1}\right\|\|P(u(\cdot))\|, \quad\left\|\mu_{2}\right\| \leq \frac{1}{\lambda} M\left\|B_{2}\right\|\|P(u(\cdot))\| .
$$


Using (13), (11), Lemmas 912 and $\left(\mathrm{H}_{2}\right)-\left(\mathrm{H}_{4}\right)$, we obtain that

$$
\begin{aligned}
\|P(u(\cdot))\| \leq & \left\|u_{a}\right\|+\left\|A^{-q}\right\|\left\{\left\|S_{\alpha}(a)\left[u_{0}-h(u(t))-A^{-p} A^{p} g(0, u(\sigma(0)))\right]\right\|_{q}\right. \\
& +\left\|A^{-p} A^{p} g(t, u(\sigma(t)))\right\|_{q} \\
& +\left\|\int_{0}^{a}(a-s)^{\alpha-1} A^{1-p} T_{\alpha}(a-s) A^{p} g(s, u(\sigma(s))) d s\right\|_{q} \\
& \left.+\left\|\int_{0}^{a}(a-s)^{\alpha-1} T_{\alpha}(a-s) v(\delta(s)) d s\right\|_{q}\right\} \\
\leq & \left\|u_{a}\right\|+\left\|A^{-q}\right\|\left\{M\left[\left\|u_{0}\right\|_{q}+k+\left\|A^{-p}\right\| L_{2}\right]+\left\|A^{-p}\right\| L_{2}\right. \\
& +\int_{0}^{a}(a-s)^{\alpha-1} \frac{\alpha M_{1-p} \Gamma(1+p)}{\Gamma(1+\alpha p)}(a-s)^{-(1-p) \alpha} L_{2} d s \\
& \left.+\int_{0}^{a}(a-s)^{\alpha-1} \frac{\alpha M_{q} \Gamma(2-q)}{\Gamma(1+\alpha(1-q))}(a-s)^{-q \alpha} \omega d s\right\} \\
\leq & \left\|u_{a}\right\|+\left\|A^{-q}\right\|\left\{M\left[\left\|u_{0}\right\|_{q}+k+\left\|A^{-p}\right\| L_{2}\right]+\left\|A^{-p}\right\| L_{2}\right. \\
& \left.+\frac{a^{p \alpha}}{p \alpha} \frac{\alpha M_{1-p} \Gamma(1+p)}{\Gamma(1+\alpha p)} L_{2}+\frac{a^{\alpha(1-q)}}{\alpha(1-q)} \frac{\alpha M_{q} \Gamma(2-q)}{\Gamma(1+\alpha(1-q))} \omega\right\} .
\end{aligned}
$$

Now, for $z \in F^{\lambda}(u)$, we have

$$
\begin{aligned}
\|z(t)\|_{q} \leq & \left\|S_{\alpha}(t)\left[B_{2} \mu_{2}(t)+u_{0}-h(u(t))-A^{-p} A^{p} g(0, u(\sigma(0)))\right]\right\|_{q} \\
& +\left\|A^{-p} A^{p} g(t, u(\sigma(t)))\right\|_{q}+\left\|\int_{0}^{t}(t-s)^{\alpha-1} A^{1-p} T_{\alpha}(t-s) A^{p} g(s, u(\sigma(s))) d s\right\|_{q} \\
& +\left\|\int_{0}^{t}(t-s)^{\alpha-1} T_{\alpha}(t-s)\left[v(\delta(s))+B_{1} \mu_{1}(s)\right] d s\right\|_{q} \\
\leq & M\left[\left\|B_{2}\right\|_{q}\left\|\mu_{2}\right\|+\left\|u_{0}\right\|_{q}+k+M_{p} L_{2}\right]+M_{p} L_{2} \\
& +\int_{0}^{t}(t-s)^{\alpha-1} \frac{\alpha M_{1-p} \Gamma(1+p)}{\Gamma(1+\alpha p)}(t-s)^{-(1-p) \alpha} L_{2} d s \\
& +\int_{0}^{t}(t-s)^{\alpha-1} \frac{\alpha M_{q} \Gamma(2-q)}{\Gamma(1+\alpha(1-q))}(t-s)^{-q \alpha}\left[\omega+\left\|B_{1}\right\|\left\|\mu_{1}\right\|\right] d s \\
\leq & M\left[\left\|B_{2}\right\|_{q}\left\|\mu_{2}\right\|+\left\|u_{0}\right\|_{q}+k+\left\|A^{-p}\right\| L_{2}\right]+\left\|A^{-p}\right\| L_{2} \\
& +\frac{a^{p \alpha}}{p \alpha} \frac{\alpha M_{1-p} \Gamma(1+p)}{\Gamma(1+\alpha p)} L_{2}+\frac{a^{\alpha(1-q)}}{\alpha(1-q)} \frac{\alpha M_{q} \Gamma(2-q)}{\Gamma(1+\alpha(1-q))}\left[\omega+\left\|B_{1}\right\|\left\|\mu_{1}\right\|\right] .
\end{aligned}
$$

Thus, for every $u \in \Omega$, there exists a positive constant $r$ satisfying $\|u\|_{\Omega} \leq r$. Hence, $F^{\lambda}\left(\Omega_{r}\right) \subset \Omega_{r}$, where $\Omega_{r}=\left\{u \in \Omega:\|u\|_{\Omega} \leq r\right\}$.

Step 2. $F^{\lambda}(u)$ is convex for each $u \in \Omega_{r}$. If $z_{1}, z_{2} \in F^{\lambda}(u)$, then there exists $v_{1}, v_{2} \in S_{f, \mu_{1}}$ such that, for each $t \in J$, we have

$$
\begin{aligned}
z_{i}(t)=S_{\alpha}(t)\left[B_{2} \mu_{2, i}(t)+u_{0}-h(u(t))-g(0, u(\sigma(0)))\right]+g(t, u(\sigma(t))) & \\
& +\int_{0}^{t}(t-s)^{\alpha-1}\left\{A T_{\alpha}(t-s) g(s, u(\sigma(s)))+T_{\alpha}(t-s)\left[v_{i}(\delta(s))+B_{1} \mu_{1, i}(s)\right]\right\} d s,
\end{aligned}
$$


where

$$
\begin{aligned}
\mu_{1, i}=B_{1}^{*} T_{\alpha}^{*}(a-t) \mathcal{R}\left(\lambda, \Gamma_{0,1}^{a}\right) & {\left[u_{a}-S_{\alpha}(a)\left[u_{0}-h(u(t))-g(0, u(\sigma(0)))\right]-g(t, u(\sigma(t)))\right.} \\
& \left.-\int_{0}^{a}(a-s)^{\alpha-1}\left\{A T_{\alpha}(a-s) g(s, u(\sigma(s)))+T_{\alpha}(a-s) v_{i}(\delta(s))\right\} d s\right], \\
\mu_{2, i}=B_{2}^{*} S_{\alpha}^{*}(a) \mathcal{R}\left(\lambda, \Gamma_{0,2}^{a}\right) & {\left[u_{a}-S_{\alpha}(a)\left[u_{0}-h(u(t))-g(0, u(\sigma(0)))\right]-g(t, u(\sigma(t)))\right.} \\
& \left.-\int_{0}^{a}(a-s)^{\alpha-1}\left\{A T_{\alpha}(a-s) g(s, u(\sigma(s)))+T_{\alpha}(a-s) v_{i}(\delta(s))\right\} d s\right],
\end{aligned}
$$

$i=1,2$. Let $0 \leq \beta \leq 1$. Then,

$$
\begin{aligned}
\beta z_{1}(t)+(1-\beta) z_{2}(t)=S_{\alpha}( & t)\left\{B_{2}\left[\beta \mu_{2,1}(t)+(1-\beta) \mu_{2,2}(t)\right]+u_{0}-h(u(t))-g(0, u(\sigma(0)))\right\} \\
+ & g(t, u(\sigma(t)))+\int_{0}^{t}(t-s)^{\alpha-1} A T_{\alpha}(t-s) g(s, u(\sigma(s))) d s \\
+ & \int_{0}^{t}(t-s)^{\alpha-1} T_{\alpha}(t-s)\left[\beta v_{1}(\delta(s))+(1-\beta) v_{2}(\delta(s))\right] d s \\
+ & \int_{0}^{t}(t-s)^{\alpha-1} T_{\alpha}(t-s) B_{1}\left[\beta \mu_{1,1}(s)+(1-\beta) \mu_{1,2}(s)\right] d s,
\end{aligned}
$$

where

$$
\begin{aligned}
\beta \mu_{1,1}( & t)+(1-\beta) \mu_{1,2}(t) \\
= & B_{1}^{*} T_{\alpha}^{*}(a-t) \mathcal{R}\left(\lambda, \Gamma_{0,1}^{a}\right)\left[u_{a}-S_{\alpha}(a)\left[u_{0}-h(u(t))-g(0, u(\sigma(0)))\right]-g(t, u(\sigma(t)))\right. \\
- & \left.\int_{0}^{a}(a-s)^{\alpha-1}\left\{A T_{\alpha}(a-s) g(s, u(\sigma(s)))+T_{\alpha}(a-s)\left[\beta v_{1}(\delta(s))+(1-\beta) v_{2}(\delta(s))\right]\right\} d s\right], \\
\beta \mu_{2,1}( & t)+(1-\beta) \mu_{2,2}(t) \\
= & B_{2}^{*} S_{\alpha}^{*}(a) \mathcal{R}\left(\lambda, \Gamma_{0,2}^{a}\right)\left[u_{a}-S_{\alpha}(a)\left[u_{0}-h(u(t))-g(0, u(\sigma(0)))\right]-g(t, u(\sigma(t)))\right. \\
- & \left.\int_{0}^{a}(a-s)^{\alpha-1}\left\{A T_{\alpha}(a-s) g(s, u(\sigma(s)))+T_{\alpha}(a-s)\left[\beta v_{1}(\delta(s))+(1-\beta) v_{2}(\delta(s))\right]\right\} d s\right] .
\end{aligned}
$$

Since the multi-valued map $f$ has convex values, then $S_{f, \mu_{1}}$ is convex. As required, we conclude that $\beta z_{1}(t)+(1-\beta) z_{2}(t) \in F^{\lambda}(u)$.

Step 3. $F^{\lambda}(u)$ is closed for each $u \in \Omega_{r}$. Let $\left\{z_{n}\right\}_{n \geq 0} \in F^{\lambda}(u)$ for $z_{n} \rightarrow z \in \Omega_{r}$. Then, there exists $v_{n} \in S_{f, \mu_{1}}$ such that

$$
\begin{aligned}
z_{n}(t)=S_{\alpha}(t)[ & \left.B_{2} \mu_{2, n}(t)+u_{0}-h(u(t))-g(0, u(\sigma(0)))\right]+g(t, u(\sigma(t))) \\
& +\int_{0}^{t}(t-s)^{\alpha-1}\left\{A T_{\alpha}(t-s) g(s, u(\sigma(s)))+T_{\alpha}(t-s)\left[v_{n}(\delta(s))+B_{1} \mu_{1, n}(s)\right]\right\} d s
\end{aligned}
$$

for every $t \in J$, where

$$
\begin{aligned}
\mu_{1, n}=B_{1}^{*} T_{\alpha}^{*}(a-t) \mathcal{R}(\lambda, & \left.\Gamma_{0,1}^{a}\right)\left[u_{a}-S_{\alpha}(a)\left[u_{0}-h(u(t))-g(0, u(\sigma(0)))\right]-g(t, u(\sigma(t)))\right. \\
& \left.-\int_{0}^{a}(a-s)^{\alpha-1}\left\{A T_{\alpha}(a-s) g(s, u(\sigma(s)))+T_{\alpha}(a-s) v_{n}(\delta(s))\right\} d s\right],
\end{aligned}
$$




$$
\begin{aligned}
\mu_{2, n}=B_{2}^{*} S_{\alpha}^{*}(a) \mathcal{R}\left(\lambda, \Gamma_{0,2}^{a}\right) & {\left[u_{a}-S_{\alpha}(a)\left[u_{0}-h(u(t))-g(0, u(\sigma(0)))\right]-g(t, u(\sigma(t)))\right.} \\
& \left.-\int_{0}^{a}(a-s)^{\alpha-1}\left\{A T_{\alpha}(a-s) g(s, u(\sigma(s)))+T_{\alpha}(a-s) v_{n}(\delta(s))\right\} d s\right] .
\end{aligned}
$$

From [37, we deduce that $S_{f, \mu_{1}}$ is weakly compact in $L^{1}(J, H)$, which implies that $v_{n}$ converges

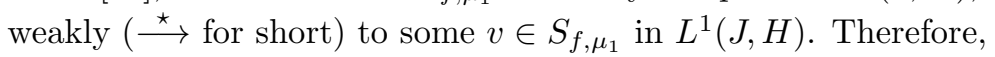

$$
\begin{array}{r}
\mu_{1, n} \stackrel{\star}{\longrightarrow} \mu_{1}=B_{1}^{*} T_{\alpha}^{*}(a-t) \\
-\int_{0}^{a}\left(\lambda, \Gamma_{0,1}^{a}\right)\left[u_{a}-S_{\alpha}(a)\left[u_{0}-h(u(t))-g(0, u(\sigma(0)))\right]-g(t, u(\sigma(t)))\right. \\
\left.\mu_{2, n} \stackrel{\star}{\longrightarrow} \mu_{2}=B_{2}^{*} S_{\alpha}^{*}(a) \mathcal{R}\left(\lambda, T_{\alpha}(a-s) g(s, u(\sigma(s)))+T_{\alpha, 2}(a-s) v(\delta(s))\right\} d s\right], \\
\left.-\int_{0}^{a}(a-s)^{\alpha-1}\left\{A T_{\alpha}(a-s) g(s, u(\sigma(s)))+T_{\alpha}(a-s) v(\delta(s))\right\} d s\right] .
\end{array}
$$

Thus,

$$
\begin{aligned}
z_{n}(t) \rightarrow z(t)= & S_{\alpha}(t)\left[B_{2} \mu_{2}(t)+u_{0}-h(u(t))-g(0, u(\sigma(0)))\right]+g(t, u(\sigma(t))) \\
& +\int_{0}^{t}(t-s)^{\alpha-1}\left\{A T_{\alpha}(t-s) g(s, u(\sigma(s)))+T_{\alpha}(t-s)\left[v(\delta(s))+B_{1} \mu_{1}(s)\right]\right\} d s .
\end{aligned}
$$

Hence, $z \in F^{\lambda}(u)$.

Step 4. $F^{\lambda}(u)$ is u.s.c. and condensing. We make the decomposition $F^{\lambda}=F_{1}^{\lambda}+F_{2}^{\lambda}$, where the operators $F_{1}^{\lambda}$ and $F_{2}^{\lambda}$ are defined by

$$
\left(F_{1}^{\lambda} u\right)(t)=-S_{\alpha}(t) g(0, u(\sigma(0)))+g(t, u(\sigma(t)))+\int_{0}^{t}(t-s)^{\alpha-1} A T_{\alpha}(t-s) g(s, u(\sigma(s))) d s
$$

and

$$
\begin{aligned}
F_{2}^{\lambda}(u)=\left\{z \in \Omega_{r}: z(t)=S_{\alpha}(t)\right. & {\left[B_{2} \mu_{2}(t)+u_{0}-h(u(t))\right] } \\
+ & \left.\int_{0}^{t}(t-s)^{\alpha-1} T_{\alpha}(t-s)\left[v(\delta(s))+B_{1} \mu_{1}(s)\right] d s, \quad v \in S_{f, \mu_{1}}\right\} .
\end{aligned}
$$

We show that $F_{1}^{\lambda}$ is a contraction operator while $F_{2}^{\lambda}$ is completely continuous. Let $u_{1}, u_{2} \in \Omega_{r}$. Then, for each $t \in J$, condition (10) gives

$$
\begin{aligned}
\left\|F_{1}^{\lambda} u_{1}(t)-F_{1}^{\lambda} u_{2}(t)\right\|_{q} \leq & \left\|S_{\alpha}(t) A^{-p}\left[A^{p} g\left(0, u_{1}(\sigma(0))\right)-A^{p} g\left(0, u_{2}(\sigma(0))\right)\right]\right\|_{q} \\
& +\left\|A^{-p}\left[A^{p} g\left(t, u_{1}(\sigma(t))\right)-A^{p} g\left(t, u_{2}(\sigma(t))\right)\right]\right\|_{q} \\
& +\left\|\int_{0}^{t}(t-s)^{\alpha-1} A^{1-p} T_{\alpha}(t-s) A^{p}\left[g\left(s, u_{1}(\sigma(s))\right)-g\left(s, u_{2}(\sigma(s))\right)\right] d s\right\|_{q} \\
& \leq\left[M\left\|A^{-p}\right\| L_{1}+\left\|A^{-p}\right\| L_{1}\right] \sup _{s \in J}\left\|u_{1}(s)-u_{2}(s)\right\|_{q} \\
& +\int_{0}^{t}(t-s)^{\alpha-1} \frac{\alpha M_{1-p} \Gamma(1+p)}{\Gamma(1+\alpha p)}(t-s)^{-(1-p) \alpha} d s L_{1} \sup _{s \in J}\left\|u_{1}(s)-u_{2}(s)\right\|_{q} \\
& \leq L_{1}\left[M\left\|A^{-p}\right\|+\left\|A^{-p}\right\|+\frac{a^{p \alpha}}{p \alpha} \frac{\alpha M_{1-p} \Gamma(1+p)}{\Gamma(1+\alpha p)}\right] \sup _{s \in J}\left\|u_{1}(s)-u_{2}(s)\right\|_{q} .
\end{aligned}
$$


Therefore, $\left\|F_{1}^{\lambda} u_{1}(t)-F_{1}^{\lambda} u_{2}(t)\right\|_{q} \leq K \sup _{s \in J}\left\|u_{1}(s)-u_{2}(s)\right\|_{q}$, where $0 \leq K<1$. Hence $F_{1}^{\lambda}$ is a contraction operator. Next, we show that $F_{2}^{\lambda}$ is u.s.c. and completely continuous. We begin to prove that $F_{2}^{\lambda}$ is completely continuous.

1. $F_{2}^{\lambda}$ is already bounded.

2. $F_{2}^{\lambda}$ is equicontinuous on $\Omega_{r}$. Let $u \in \Omega_{r}, z \in\left(F_{2}^{\lambda}\right)(u)$. Then there exists $v \in S_{f, \mu_{1}}$ such that

$$
z(t)=S_{\alpha}(t)\left[B_{2} \mu_{2}(t)+u_{0}-h(u(t))\right]+\int_{0}^{t}(t-s)^{\alpha-1} T_{\alpha}(t-s)\left[v(\delta(s))+B_{1} \mu_{1}(s)\right] d s
$$

for each $t \in J$. It follows that

$$
\begin{aligned}
\|z(\tau)-z(0)\|_{q}= & \|\left(S_{\alpha}(\tau)-I\right)\left[A^{q} B_{2} \mu_{2}(t)+A^{q} u_{0}-A^{q} h(u(t))\right] \\
& +\int_{0}^{\tau}(\tau-s)^{\alpha-1} A^{q} T_{\alpha}(\tau-s)\left[v(\delta(s))+B_{1} \mu_{1}(s)\right] d s \| \\
\leq & \left\|\left(S_{\alpha}(\tau)-I\right)\left[A^{q} B_{2} \mu_{2}(t)+A^{q} u_{0}-A^{q} h(u(t))\right]\right\| \\
& +\frac{\tau^{\alpha(1-q)}}{\alpha(1-q)} \frac{\alpha M_{q} \Gamma(2-q)}{\Gamma(1+\alpha(1-q))}\left[\omega+\left\|B_{1}\right\|\left\|\mu_{1}\right\|\right] \longrightarrow 0
\end{aligned}
$$

as $\tau \rightarrow 0$ uniformly, since, by $\left(\mathrm{H}_{3}\right)$ and Lemma 12 the complete continuity of $A^{q} h$ and the strong continuity of $S_{\alpha}(t)$ at $t=0$ are satisfied, respectively. Let $\tau_{1}, \tau_{2} \in J$ with $0<s<\tau_{1}<\tau_{2} \leq a$. Then

$$
\begin{aligned}
\left\|z\left(\tau_{2}\right)-z\left(\tau_{1}\right)\right\|_{q} \leq & \left\|\left[S_{\alpha}\left(\tau_{2}\right)-S_{\alpha}\left(\tau_{1}\right)\right]\left[B_{2} \mu_{2}(t)+u_{0}-h(u(t))\right]\right\|_{q} \\
& +\left\|\int_{\tau_{1}}^{\tau_{2}}\left(\tau_{2}-s\right)^{\alpha-1} T_{\alpha}\left(\tau_{2}-s\right)\left[v(\delta(s))+B_{1} \mu_{1}(s)\right] d s\right\|_{q} \\
& +\left\|\int_{0}^{\tau_{1}}\left[\left(\tau_{2}-s\right)^{\alpha-1}-\left(\tau_{1}-s\right)^{\alpha-1}\right] T_{\alpha}\left(\tau_{2}-s\right)\left[v(\delta(s))+B_{1} \mu_{1}(s)\right] d s\right\|_{q} \\
& +\left\|\int_{0}^{\tau_{1}}\left(\tau_{1}-s\right)^{\alpha-1}\left[T_{\alpha}\left(\tau_{2}-s\right)-T_{\alpha}\left(\tau_{1}-s\right)\right]\left[v(\delta(s))+B_{1} \mu_{1}(s)\right] d s\right\|_{q} \\
& \leq\left\|S_{\alpha}\left(\tau_{2}\right)-S_{\alpha}\left(\tau_{1}\right)\right\|_{q}\left[\left\|B_{2}\right\|\left\|\mu_{2}(t)\right\|+\left\|u_{0}\right\|_{q}+\|h(u(t))\|\right] \\
& +\int_{\tau_{1}}^{\tau_{2}}\left(\tau_{2}-s\right)^{\alpha-1}\left\|A^{q} T_{\alpha}\left(\tau_{2}-s\right)\right\|\left[\|v(\delta(s))\|+\left\|B_{1}\right\|\left\|\mu_{1}(s)\right\|\right] d s \\
& +\int_{0}^{\tau_{1}}\left[\left(\tau_{2}-s\right)^{\alpha-1}-\left(\tau_{1}-s\right)^{\alpha-1}\right]\left\|A^{q} T_{\alpha}\left(\tau_{2}-s\right)\right\|\left[\|v(\delta(s))\|+\left\|B_{1}\right\|\left\|\mu_{1}(s)\right\|\right] d s \\
& +\int_{0}^{\tau_{1}}\left(\tau_{1}-s\right)^{\alpha-1} \| A^{q}\left[T_{\alpha}\left(\tau_{2}-s\right)-T_{\alpha}\left(\tau_{1}-s\right) \|\right]\left[\|v(\delta(s))\|+\left\|B_{1}\right\|\left\|\mu_{1}(s)\right\|\right] d s \\
& \leq\left\|S_{\alpha}\left(\tau_{2}\right)-S_{\alpha}\left(\tau_{1}\right)\right\|_{q}\left[\left\|B_{2}\right\|\left\|\mu_{2}(t)\right\|+\left\|u_{0}\right\|_{q}+\|h(u(t))\|\right] \\
& +\int_{\tau_{1}}^{\tau_{2}}\left(\tau_{2}-s\right)^{\alpha-1}\left\|A^{q} T_{\alpha}\left(\tau_{2}-s\right)\right\|\left[\omega+\left\|B_{1}\right\|\left\|\mu_{1}(s)\right\|\right] d s \\
& +\int_{0}^{\tau_{1}-\epsilon}\left[\left(\tau_{2}-s\right)^{\alpha-1}-\left(\tau_{1}-s\right)^{\alpha-1}\right]\left\|A^{q} T_{\alpha}\left(\tau_{2}-s\right)\right\|\left[\omega+\left\|B_{1}\right\|\left\|\mu_{1}(s)\right\|\right] d s \\
& +\int_{\tau_{1}-\epsilon}^{\tau_{1}}\left[\left(\tau_{2}-s\right)^{\alpha-1}-\left(\tau_{1}-s\right)^{\alpha-1}\right]\left\|A^{q} T_{\alpha}\left(\tau_{2}-s\right)\right\|\left[\omega+\left\|B_{1}\right\|\left\|\mu_{1}(s)\right\|\right] d s \\
& +\int_{0}^{\tau_{1}-\epsilon}\left(\tau_{1}-s\right)^{\alpha-1} \| A^{q}\left[T_{\alpha}\left(\tau_{2}-s\right)-T_{\alpha}\left(\tau_{1}-s\right) \|\right]\left[\omega+\left\|B_{1}\right\|\left\|\mu_{1}(s)\right\|\right] d s \\
& +\int_{\tau_{1}-\epsilon}^{\tau_{1}}\left(\tau_{1}-s\right)^{\alpha-1} \| A^{q}\left[T_{\alpha}\left(\tau_{2}-s\right)-T_{\alpha}\left(\tau_{1}-s\right) \|\right]\left[\omega+\left\|B_{1}\right\|\left\|\mu_{1}(s)\right\|\right] d s \\
&
\end{aligned}
$$




$$
\begin{aligned}
& \leq\left\|S_{\alpha}\left(\tau_{2}\right)-S_{\alpha}\left(\tau_{1}\right)\right\|_{q}\left[\left\|B_{2}\right\|\left\|\mu_{2}(t)\right\|+\left\|u_{0}\right\|_{q}+\|h(u(t))\|\right] \\
& +\frac{\alpha M_{q} \Gamma(2-q)}{\Gamma(1+\alpha(1-q))}\left[\frac{\left(\tau_{2}-\tau_{1}\right)^{\alpha(1-q)}}{\alpha(1-q)}\left[\omega+\left\|B_{1}\right\|\left\|\mu_{1}\right\|\right]\right. \\
& +\int_{0}^{\tau_{1}-\epsilon}\left[\left(\tau_{2}-s\right)^{\alpha-1}-\left(\tau_{1}-s\right)^{\alpha-1}\right]\left(\tau_{2}-s\right)^{-q \alpha}\left[\omega+\left\|B_{1}\right\|\left\|\mu_{1}(s)\right\|\right] d s \\
& +\int_{\tau_{1}-\epsilon}^{\tau_{1}}\left[\left(\tau_{2}-s\right)^{\alpha-1}-\left(\tau_{1}-s\right)^{\alpha-1}\right]\left(\tau_{2}-s\right)^{-q \alpha}\left[\omega+\left\|B_{1}\right\|\left\|\mu_{1}(s)\right\|\right] d s \\
& +\int_{0}^{\tau_{1}-\epsilon}\left(\tau_{1}-s\right)^{\alpha-1}\left[\left(\tau_{2}-s\right)^{-q \alpha}-\left(\tau_{1}-s\right)^{-q \alpha}\right]\left[\omega+\left\|B_{1}\right\|\left\|\mu_{1}(s)\right\|\right] d s \\
& \left.+\int_{\tau_{1}-\epsilon}^{\tau_{1}}\left(\tau_{1}-s\right)^{\alpha-1}\left[\left(\tau_{2}-s\right)^{-q \alpha}-\left(\tau_{1}-s\right)^{-q \alpha}\right]\left[\omega+\left\|B_{1}\right\|\left\|\mu_{1}(s)\right\|\right] d s\right] .
\end{aligned}
$$

In view of Lemma 12, $S_{\alpha}(\cdot)$ and $T_{\alpha}(\cdot)$ are compact and strongly continuous operators, which imply the continuity of those operators in the uniform operator topology on $(0, a]$. Concluding, as $\tau_{2}-\tau_{1} \rightarrow 0$, with $\epsilon$ sufficiently small, the right-hand side of the above inequality tends to zero independently of $u \in \Omega_{r}$. This shows the equicontinuity of $F_{2}^{\lambda}$ on $\Omega_{r}$.

3. $\left(F_{2}^{\lambda} \Omega_{r}\right)(t)=\left\{z(t): z \in F_{2}^{\lambda}\left(\Omega_{r}\right)\right\}$ is relatively compact in $H_{q}$ for each $t \in J$. Clearly, $\left(F_{2}^{\lambda} \Omega_{r}\right)(t)$ is relatively compact in $H_{q}$ for $t=0$. Let $0<t \leq a$ be fixed. For $u \in \Omega_{r}$ and $z \in\left(F_{2}^{\lambda}\right)(u)$, there exists a function $v \in S_{f, \mu_{1}}$ such that

$$
z(t)=S_{\alpha}(t)\left[B_{2} \mu_{2}(t)+u_{0}-h(u(t))\right]+\int_{0}^{t}(t-s)^{\alpha-1} T_{\alpha}(t-s)\left[v(\delta(s))+B_{1} \mu_{1}(s)\right] d s .
$$

For $0<\gamma<1$, we have

$$
\begin{aligned}
\left\|A^{\gamma} z(t)\right\| \leq & \left\|S_{\alpha}(t)\right\|\left\|A^{\gamma}\left[B_{2} \mu_{2}(t)+u_{0}-h(u(t))\right]\right\| \\
& +\int_{0}^{t}(t-s)^{\alpha-1}\left\|A^{\gamma} T_{\alpha}(t-s)\right\|\left\|v(\delta(s))+B_{1} \mu_{1}(s)\right\| d s \\
\leq & \left\|S_{\alpha}(t)\right\| \| A^{\gamma} A^{-q}\left[B_{2} \mu_{2}(t)+u_{0}-h(u(t)) \|_{q}\right] \\
& +\int_{0}^{t}(t-s)^{\alpha-1}\left\|A^{\gamma} A^{-q} T_{\alpha}(t-s)\right\|_{q}\left[\|v(\delta(s))\|+\left\|B_{1}\right\|\left\|\mu_{1}(s)\right\|\right] d s \\
\leq & M\left\|A^{-q \gamma}\right\|\left[\left\|B_{2}\right\|\left\|\mu_{2}\right\|_{q}+\left\|u_{0}\right\|_{q}+k\right] \\
& +\left\|A^{-q \gamma}\right\| \frac{a^{\alpha(1-q)}}{\alpha(1-q)} \frac{\alpha M_{q} \Gamma(2-q)}{\Gamma(1+\alpha(1-q))}\left[\omega+\left\|B_{1}\right\|\left\|\mu_{1}\right\|\right] .
\end{aligned}
$$

By Remark 10, $A^{-q \gamma}$ is bounded since $0<q \gamma<1$. Clearly, $A^{\gamma} F_{2}^{\lambda} u(t)$ is bounded in $H$. It is known from [38] that $A^{-\gamma}: H \rightarrow H_{q}$ is compact for $0 \leq q<\gamma<1$. Then $\left(F_{2}^{\lambda} \Omega_{r}\right)(t)$ is relatively compact in $H_{q}$ for each $t \in J$. As a consequence of Step 4.1, together with the Arzela-Ascoli theorem, we conclude that $F_{2}^{\lambda}$ is completely continuous.

4. $F_{2}^{\lambda}$ has a closed graph. From above we have that $F_{2}^{\lambda}(u)$ is a relatively compact and closed set for every $u \in \Omega_{r}$. Hence $F_{2}^{\lambda}(u)$ is a compact set. Let $u_{n} \rightarrow u_{*}, u_{n} \in \Omega_{r}, z_{n} \in F_{2}^{\lambda}\left(u_{n}\right)$ and $z_{n} \rightarrow z_{*}$. We shall prove that $z_{*} \in F_{2}^{\lambda}\left(u_{*}\right)$. Note that $z_{n} \in F_{2}^{\lambda}\left(u_{n}\right)$, which means that there exists $v_{n} \in S_{f, \mu_{1, n}}$ such that

$$
z_{n}(t)=S_{\alpha}(t)\left[B_{2} \mu_{2, n}(t)+u_{0}-h\left(u_{n}\right)\right]+\int_{0}^{t}(t-s)^{\alpha-1} T_{\alpha}(t-s)\left[v_{n}(\delta(s))+B_{1} \mu_{1, n}(s)\right] d s
$$

for each $t \in J$, where

$$
\begin{aligned}
\mu_{1, n}=B_{1}^{*} T_{\alpha}^{*}(a-t) \mathcal{R}( & \left.\lambda, \Gamma_{0,1}^{a}\right)\left[u_{a}-S_{\alpha}(a)\left[u_{0}-h\left(u_{n}\right)-g\left(0, u_{n}(\sigma(0))\right)\right]-g\left(a, u_{n}(\sigma(a))\right)\right. \\
& \left.-\int_{0}^{a}(a-s)^{\alpha-1}\left\{A T_{\alpha}(a-s) g\left(s, u_{n}(\sigma(s))\right)+T_{\alpha}(a-s) v_{n}(\delta(s))\right\} d s\right],
\end{aligned}
$$




$$
\begin{aligned}
\mu_{2, n}=B_{2}^{*} S_{\alpha}^{*}(a) \mathcal{R}\left(\lambda, \Gamma_{0,2}^{a}\right) & {\left[u_{a}-S_{\alpha}(a)\left[u_{0}-h\left(u_{n}\right)-g\left(0, u_{n}(\sigma(0))\right)\right]-g\left(a, u_{n}(\sigma(a))\right)\right.} \\
- & \left.\int_{0}^{a}(a-s)^{\alpha-1}\left\{A T_{\alpha}(a-s) g\left(s, u_{n}(\sigma(s))\right)+T_{\alpha}(a-s) v_{n}(\delta(s))\right\} d s\right] .
\end{aligned}
$$

We prove the existence of $v_{*} \in S_{f, \mu_{1, *}}$ such that

$$
z_{*}(t)=S_{\alpha}(t)\left[B_{2} \mu_{2, *}(t)+u_{0}-h\left(u_{*}\right)\right]+\int_{0}^{t}(t-s)^{\alpha-1} T_{\alpha}(t-s)\left[v_{*}(\delta(s))+B_{1} \mu_{1, *}(s)\right] d s
$$

for each $t \in J$, where

$$
\begin{aligned}
\mu_{1, *}=B_{1}^{*} T_{\alpha}^{*}(a-t) \mathcal{R}\left(\lambda, \Gamma_{0,1}^{a}\right)\left[u_{a}-S_{\alpha}(a)\left[u_{0}-h\left(u_{*}\right)-g\left(0, u_{*}(\sigma(0))\right)\right]-g\left(a, u_{*}(\sigma(a))\right)\right. & \\
- & \left.\int_{0}^{a}(a-s)^{\alpha-1}\left\{A T_{\alpha}(a-s) g\left(s, u_{*}(\sigma(s))\right)+T_{\alpha}(a-s) v_{*}(\delta(s))\right\} d s\right], \\
\mu_{2, *}=B_{2}^{*} S_{\alpha}^{*}(a) \mathcal{R}\left(\lambda, \Gamma_{0,2}^{a}\right) & {\left[u_{a}-S_{\alpha}(a)\left[u_{0}-h\left(u_{*}\right)-g\left(0, u_{*}(\sigma(0))\right)\right]-g\left(a, u_{*}(\sigma(a))\right)\right.} \\
- & \left.\int_{0}^{a}(a-s)^{\alpha-1}\left\{A T_{\alpha}(a-s) g\left(s, u_{*}(\sigma(s))\right)+T_{\alpha}(a-s) v_{*}(\delta(s))\right\} d s\right] .
\end{aligned}
$$

Consider the linear continuous operator $P: L^{1}\left(J^{\prime}, H\right) \rightarrow L^{2}\left(J^{\prime}, H\right)$ defined by

$$
\begin{aligned}
v \rightarrow P(v)(t)= & \int_{0}^{t}(t-s)^{\alpha-1} T_{\alpha}(t-s) \\
& \times\left[v(\delta(s))-B_{1} B_{1}^{*} T_{\alpha}^{*}(a-s) \mathcal{R}\left(\lambda, \Gamma_{0,1}^{a}\right) \int_{0}^{a}(a-\eta)^{\alpha-1} T_{\alpha}(a-\eta) v(\delta(\eta)) d \eta\right] d s .
\end{aligned}
$$

By (14) we get

$$
\begin{gathered}
z_{n}(t)-S_{\alpha}(t)\left[B_{2} \mu_{2, n}(t)+u_{0}-h\left(u_{n}\right)\right]-\int_{0}^{t}(t-s)^{\alpha-1} T_{\alpha}(t-s) B_{1} B_{1}^{*} T_{\alpha}^{*}(a-s) \mathcal{R}\left(\lambda, \Gamma_{0,1}^{a}\right) \\
\times\left\{u_{a}-S_{\alpha}(a)\left[u_{0}-h\left(u_{n}\right)-g\left(0, u_{n}(\sigma(0))\right)\right]-g\left(a, u_{n}(\sigma(a))\right)\right. \\
\left.\quad-\int_{0}^{a}(a-\eta)^{\alpha-1} A T_{\alpha}(a-\eta) g\left(\eta, u_{n}(\sigma(\eta))\right) d \eta\right\} d s \in P\left(S_{f, \mu_{1, n}}\right),
\end{gathered}
$$

which converges to

$$
\begin{aligned}
z_{*}(t)-S_{\alpha}(t)\left[B_{2} \mu_{2, *}(t)+u_{0}-h\left(u_{*}\right)\right]-\int_{0}^{t}(t-s)^{\alpha-1} T_{\alpha}(t-s) B_{1} B_{1}^{*} T_{\alpha}^{*}(a-s) \mathcal{R}\left(\lambda, \Gamma_{0,1}^{a}\right) \\
\times\left\{u_{a}-S_{\alpha}(a)\left[u_{0}-h\left(u_{*}\right)-g\left(0, u_{*}(\sigma(0))\right)\right]-g\left(a, u_{*}(\sigma(a))\right)\right. \\
\left.\quad-\int_{0}^{a}(a-\eta)^{\alpha-1} A T_{\alpha}(a-\eta) g\left(\eta, u_{*}(\sigma(\eta))\right) d \eta\right\} d s
\end{aligned}
$$

in $\Omega_{r}$, uniformly as $n \rightarrow \infty$. From Proposition 7 , it follows that $P \circ S_{f}$ is a closed graph operator. Hence we have that

$$
\begin{gathered}
z_{*}(t)-S_{\alpha}(t)\left[B_{2} \mu_{2, *}(t)+u_{0}-h\left(u_{*}\right)\right]-\int_{0}^{t}(t-s)^{\alpha-1} T_{\alpha}(t-s) B_{1} B_{1}^{*} T_{\alpha}^{*}(a-s) \mathcal{R}\left(\lambda, \Gamma_{0,1}^{a}\right) \\
\times\left\{u_{a}-S_{\alpha}(a)\left[u_{0}-h\left(u_{*}\right)-g\left(0, u_{*}(\sigma(0))\right)\right]-g\left(a, u_{*}(\sigma(a))\right)\right. \\
\left.\quad-\int_{0}^{a}(a-\eta)^{\alpha-1} A T_{\alpha}(a-\eta) g\left(\eta, u_{*}(\sigma(\eta))\right) d \eta\right\} d s \in P\left(S_{f, \mu_{1, *}}\right),
\end{gathered}
$$


that is, there exists $v_{*}(t) \in S_{f, \mu_{1, *}}$ such that

$$
\begin{aligned}
P\left(v_{*}(t)\right)= & z_{*}(t)-S_{\alpha}(t)\left[B_{2} \mu_{2, *}(t)+u_{0}-h\left(u_{*}\right)\right] \\
& -\int_{0}^{t}(t-s)^{\alpha-1} T_{\alpha}(t-s) B_{1} B_{1}^{*} T_{\alpha}^{*}(a-s) \mathcal{R}\left(\lambda, \Gamma_{0,1}^{a}\right) \\
& \times\left\{u_{a}-S_{\alpha}(a)\left[u_{0}-h\left(u_{*}\right)-g\left(0, u_{*}(\sigma(0))\right)\right]-g\left(a, u_{*}(\sigma(a))\right)\right. \\
& \left.-\int_{0}^{a}(a-\eta)^{\alpha-1} A T_{\alpha}(a-\eta) g\left(\eta, u_{*}(\sigma(\eta))\right) d \eta\right\} d s \\
= & \int_{0}^{t}(t-s)^{\alpha-1} T_{\alpha}(t-s) \\
& \times\left[v_{*}(\delta(s))-B_{1} B_{1}^{*} T_{\alpha}^{*}(a-s) \mathcal{R}\left(\lambda, \Gamma_{0,1}^{a}\right) \int_{0}^{a}(a-\eta)^{\alpha-1} T_{\alpha}(a-\eta) v_{*}(\delta(\eta)) d \eta\right] d s .
\end{aligned}
$$

This shows that $z_{*} \in F_{2}^{\lambda}\left(u_{*}\right)$. Therefore, $F_{2}^{\lambda}$ has a closed graph and $F_{2}^{\lambda}$ is a completely continuous multi-valued map with compact value. Thus, $F_{2}^{\lambda}$ is u.s.c. On the other hand, $F_{1}^{\lambda}$ is proved a contraction operator and hence $F^{\lambda}=F_{1}^{\lambda}+F_{2}^{\lambda}$ is u.s.c. and condensing. According to Lemma 8 we ensure the existence of a fixed point $u^{\lambda}(\cdot)$ for $F^{\lambda}$ in $\Omega_{r}$.

Theorem 16. If $\left(H_{1}\right)-\left(H_{4}\right)$ are satisfied and $\lambda \mathcal{R}\left(\lambda, \Gamma_{0, i}^{a}\right) \rightarrow 0$ in the strong operator topology as $\lambda \rightarrow 0^{+}, i=1,2$, then the nonlocal-control fractional delay system (11) -(2) is approximately controllable on $J$.

Proof. According to Theorem 15] $F^{\lambda}$ has a fixed point in $\Omega_{r}$ for any $\lambda \in(0,1)$. This implies that there exists $\bar{u}^{\lambda} \in F^{\lambda}\left(\bar{u}^{\lambda}\right)$, that is, there is $\bar{v}^{\lambda} \in S_{f, \bar{\mu}_{1} \lambda}$ such that

$$
\begin{aligned}
& \bar{u}^{\lambda}(t)=S_{\alpha}(t) {\left[B_{2}{\overline{\mu_{2}}}^{\lambda}(t)+u_{0}-h\left(\bar{u}^{\lambda}\right)-g\left(0, \bar{u}^{\lambda}(\sigma(0))\right)\right]+g\left(t, \bar{u}^{\lambda}(\sigma(t))\right) } \\
&+\int_{0}^{t}(t-s)^{\alpha-1}\left\{A T_{\alpha}(t-s) g\left(s, \bar{u}^{\lambda}(\sigma(s))\right)+T_{\alpha}(t-s)\left[\bar{v}^{\lambda}(\delta(s))+B_{1} \bar{\mu}_{1}^{\lambda}(s)\right]\right\} d s
\end{aligned}
$$

where

$$
\begin{array}{r}
{\overline{\mu_{1}}}^{\lambda}(t)=B_{1}^{*} T_{\alpha}^{*}(a-t) \mathcal{R}\left(\lambda, \Gamma_{0,1}^{a}\right)\left[u_{a}-S_{\alpha}(a)\left[u_{0}-h\left(\bar{u}^{\lambda}(t)\right)-g\left(0, \bar{u}^{\lambda}(\sigma(0))\right)\right]-g\left(t, \bar{u}^{\lambda}(\sigma(t))\right)\right. \\
\left.-\int_{0}^{a}(a-s)^{\alpha-1}\left\{A T_{\alpha}(a-s) g\left(s, \bar{u}^{\lambda}(\sigma(s))\right)+T_{\alpha}(a-s) \bar{v}^{\lambda}(\delta(s))\right\} d s\right], \\
{\overline{\mu_{2}}}^{\lambda}(t)=B_{2}^{*} S_{\alpha}^{*}(a) \mathcal{R}\left(\lambda, \Gamma_{0,2}^{a}\right)\left[u_{a}-S_{\alpha}(a)\left[u_{0}-h\left(\bar{u}^{\lambda}(t)\right)-g\left(0, \bar{u}^{\lambda}(\sigma(0))\right)\right]-g\left(t, \bar{u}^{\lambda}(\sigma(t))\right)\right. \\
\left.-\int_{0}^{a}(a-s)^{\alpha-1}\left\{A T_{\alpha}(a-s) g\left(s, \bar{u}^{\lambda}(\sigma(s))\right)+T_{\alpha}(a-s) \bar{v}^{\lambda}(\delta(s))\right\} d s\right] .
\end{array}
$$

Now,

$$
\begin{aligned}
\bar{u}^{\lambda}(a)=S_{\alpha}(a)\left[B_{2}{\overline{\mu_{2}}}^{\lambda}(a)+u_{0}-h\left(\bar{u}^{\lambda}\right)-g\left(0, \bar{u}^{\lambda}(\sigma(0))\right)\right]+g\left(a, \bar{u}^{\lambda}(\sigma(a))\right) \\
+\int_{0}^{a}(a-s)^{\alpha-1}\left\{A T_{\alpha}(a-s) g\left(s, \bar{u}^{\lambda}(\sigma(s))\right)+T_{\alpha}(a-s)\left[\bar{v}^{\lambda}(\delta(s))+B_{1} \bar{\mu}_{1}^{\lambda}(s)\right]\right\} d s
\end{aligned}
$$


and

$$
\begin{aligned}
u_{a}-\bar{u}^{\lambda}(a)=u_{a} & -\Gamma_{0,2}^{a} \mathcal{R}\left(\lambda, \Gamma_{0,2}^{a}\right)\left\{u_{a}-S_{\alpha}(a)\left[u_{0}-h\left(\bar{u}^{\lambda}\right)-g\left(0, \bar{u}^{\lambda}(\sigma(0))\right)\right]-g\left(a, \bar{u}^{\lambda}(\sigma(a))\right)\right. \\
& \left.-\int_{0}^{a}(a-s)^{\alpha-1}\left\{A T_{\alpha}(a-s) g\left(s, \bar{u}^{\lambda}(\sigma(s))\right)+T_{\alpha}(a-s) \bar{v}^{\lambda}(\delta(s))\right\} d s\right\} \\
& -S_{\alpha}(a)\left[u_{0}-h\left(\bar{u}^{\lambda}\right)-g\left(0, \bar{u}^{\lambda}(\sigma(0))\right)\right]-g\left(a, \bar{u}^{\lambda}(\sigma(a))\right) \\
& -\int_{0}^{a}(a-s)^{\alpha-1}\left\{A T_{\alpha}(a-s) g\left(s, \bar{u}^{\lambda}(\sigma(s))\right)+T_{\alpha}(a-s) \bar{v}^{\lambda}(\delta(s))\right\} d s, \\
& -\Gamma_{0,1}^{a} \mathcal{R}\left(\lambda, \Gamma_{0,1}^{a}\right)\left\{u_{a}-S_{\alpha}(a)\left[u_{0}-h\left(\bar{u}^{\lambda}\right)-g\left(0, \bar{u}^{\lambda}(\sigma(0))\right)\right]-g\left(a, \bar{u}^{\lambda}(\sigma(a))\right)\right. \\
& \left.-\int_{0}^{a}(a-s)^{\alpha-1}\left\{A T_{\alpha}(a-s) g\left(s, \bar{u}^{\lambda}(\sigma(s))\right)+T_{\alpha}(a-s) \bar{v}^{\lambda}(\delta(s))\right\} d s\right\} .
\end{aligned}
$$

From (9) we have $I-\Gamma_{0, i}^{a} \mathcal{R}\left(\lambda, \Gamma_{0, i}^{a}\right)=\lambda \mathcal{R}\left(\lambda, \Gamma_{0, i}^{a}\right), i=1,2$, and we deduce that

$$
\begin{aligned}
& u_{a}-\bar{u}^{\lambda}(a)=\lambda\left[\mathcal{R}\left(\lambda, \Gamma_{0,1}^{a}\right)+\mathcal{R}\left(\lambda, \Gamma_{0,2}^{a}\right)\right]\left\{u_{a}-S_{\alpha}(a)\left[u_{0}-h\left(\bar{u}^{\lambda}\right)-g\left(0, \bar{u}^{\lambda}(\sigma(0))\right)\right]\right. \\
& \left.-g\left(a, \bar{u}^{\lambda}(\sigma(a))\right)-\int_{0}^{a}(a-s)^{\alpha-1}\left\{A T_{\alpha}(a-s) g\left(s, \bar{u}^{\lambda}(\sigma(s))\right)+T_{\alpha}(a-s) \bar{v}^{\lambda}(\delta(s))\right\} d s\right\} .
\end{aligned}
$$

According to the compactness of $S_{\alpha}(t), t>0$, and the uniform boundedness of $h$ and $g$, we see that there is a subsequence of $S_{\alpha}(a)\left[h\left(\bar{u}^{\lambda}\right)-g\left(0, \bar{u}^{\lambda}(\sigma(0))\right)\right]$ that converges to some $u_{1}$ as $\lambda \rightarrow 0^{+}$. By assumption $\left(\mathrm{H}_{1}\right)$, we can choose a sufficiently small positive constant $\epsilon>0, q+\epsilon<1$, such that $A^{q+\epsilon} g\left(a, \bar{u}^{\lambda}(\sigma(a))\right)$ is bounded in $H$. Since $g\left(a, \bar{u}^{\lambda}(\sigma(a))\right)=A^{-(q+\epsilon)} A^{q+\epsilon} g\left(a, \bar{u}^{\lambda}(\sigma(a))\right)$, $g\left(a, \bar{u}^{\lambda}(\sigma(a))\right)$ is clearly relatively compact in $H_{q}$ and hence in $H\left(A^{-(q+\epsilon)}: H \rightarrow H_{q}\right.$ is compact). It means that there is $u_{2} \in H$ such that $g\left(a, \bar{u}^{\lambda}(\sigma(a))\right) \rightarrow u_{2}$ in $\|\cdot\|$ as $\lambda \rightarrow 0^{+}$(here $g$ is a subsequence of itself). On the other hand,

$$
\int_{0}^{a}(a-s)^{\alpha-1} A T_{\alpha}(a-s) g\left(s, \bar{u}^{\lambda}(\sigma(s))\right) d s=\int_{0}^{a}(a-s)^{\alpha-1} A^{1-q} T_{\alpha}(a-s) A^{q} g\left(s, \bar{u}^{\lambda}(\sigma(s))\right) d s
$$

and, by $\left(\mathrm{H}_{1}\right), A^{q} g\left(s, \bar{u}^{\lambda}(\sigma(s))\right) \in L^{2}(J, H)$. Then we can get a subsequence, still denoted by $A^{q} g\left(s, \bar{u}^{\lambda}(\sigma(s))\right)$, which converges weakly to some $g(s) \in L^{2}(J, H)$. Similarly as the proof of the compactness of $F_{2}^{\lambda}$ in Theorem [15, it is easy to see that the mapping

$$
u(t) \rightarrow \int_{0}^{t}(t-s)^{\alpha-1} A^{1-q} T_{\alpha}(t-s) u(s) d s,
$$

from $L^{2}(J, H)$ to $C(J, H)$, is compact. Then,

$$
\int_{0}^{a}(a-s)^{\alpha-1} A^{1-q} T_{\alpha}(a-s)\left[A^{q} g\left(s, \bar{u}^{\lambda}(\sigma(s))\right)-g(s)\right] d s \rightarrow 0
$$

as $\lambda \rightarrow 0^{+}$. Similarly, $\bar{v}^{\lambda}(\delta(s))$ is uniformly bounded in $L^{2}\left(J^{\prime}, H\right)$ and so converges weakly to some $v(s) \in L^{2}(J, H)$. The mapping

$$
u(t) \rightarrow \int_{0}^{t}(t-s)^{\alpha-1} T_{\alpha}(t-s) u(s) d s
$$

is also compact on $L^{2}(J, H)$. It follows that

$$
\int_{0}^{a}(a-s)^{\alpha-1} T_{\alpha}(a-s)\left[\bar{v}^{\lambda}(s)-v(s)\right] d s \rightarrow 0
$$


as $\lambda \rightarrow 0^{+}$. Using (15), we get

$$
\begin{aligned}
\left\|u_{a}-\bar{u}^{\lambda}(a)\right\|= & \| \lambda\left[\mathcal{R}\left(\lambda, \Gamma_{0,1}^{a}\right)+\mathcal{R}\left(\lambda, \Gamma_{0,2}^{a}\right)\right]\left\{u_{a}-S_{\alpha}(a)\left[u_{0}-h\left(\bar{u}^{\lambda}\right)-g\left(0, \bar{u}^{\lambda}(\sigma(0))\right)\right]\right. \\
& \left.-g\left(a, \bar{u}^{\lambda}(\sigma(a))\right)+T_{\alpha}(a-s) \bar{v}^{\lambda}(\delta(s))\right\} \\
& -\int_{0}^{a}(a-s)^{\alpha-1}\left\{A T_{\alpha}(a-s) g\left(s, \bar{u}^{\lambda}(\sigma(s))\right)\right\} d s \| \\
\leq & \| \lambda\left[\mathcal{R}\left(\lambda, \Gamma_{0,1}^{a}\right)+\mathcal{R}\left(\lambda, \Gamma_{0,2}^{a}\right)\right]\left\{u_{a}-S_{\alpha}(a)\left[u_{0}-u_{1}\right]-u_{2}\right. \\
& \left.-\int_{0}^{a}(a-s)^{\alpha-1}\left\{A^{q} T_{\alpha}(a-s) g(s)+T_{\alpha}(a-s) v(\delta(s))\right\} d s\right\} \| \\
& +\left\|\lambda\left[\mathcal{R}\left(\lambda, \Gamma_{0,1}^{a}\right)+\mathcal{R}\left(\lambda, \Gamma_{0,2}^{a}\right)\right]\right\|\left\{\left\|S_{\alpha}(a)\left[h\left(\bar{u}^{\lambda}\right)-g\left(0, \bar{u}^{\lambda}(\sigma(0))\right)-u_{1}\right]\right\|\right. \\
& +\| \int_{0}^{a}(a-s)^{\alpha-1}\left\{A^{1-q} T_{\alpha}(a-s)\left[A^{q} g\left(s, \bar{u}^{\lambda}(\sigma(s))\right)-g(s)\right]\right. \\
& \left.\left.+\left\|g\left(a, \bar{u}^{\lambda}(\sigma(a))\right)-u_{2}\right\|+T_{\alpha}(a-s)\left[\bar{v}^{\lambda}(\delta(s))-v(s)\right]\right\} d s \|\right\} .
\end{aligned}
$$

Moreover, by the assumption that we have $\lambda \mathcal{R}\left(\lambda, \Gamma_{0, i}^{a}\right) \rightarrow 0$ in the strong operator topology as $\lambda \rightarrow 0^{+}, i=1,2$, we ensure that $\left\|u_{a}-\bar{u}^{\lambda}(a)\right\| \rightarrow 0$ as $\lambda \rightarrow 0^{+}$. Therefore, the fractional dynamic inclusion (10)-(2) is approximately controllable on $J$.

\section{An Example}

In this section, we apply Theorems 15 and 16 to the following fractional partial functional differential inclusion with nonlocal control condition:

$$
\begin{gathered}
\frac{\partial^{\alpha}}{\partial t^{\alpha}}[u(x, t)-x \arctan u(x, \sin t)] \in \frac{\partial^{2} u(x, t)}{\partial x^{2}}+\int_{0}^{t} b(t, s) \exp \xi(x, \sin s) d s, \\
u(x, 0)-u_{0}(x)=\sum_{k=1}^{m} c_{k}\left[\xi\left(x, t_{k}\right)-u\left(x, t_{k}\right)\right], \quad x \in[0, \pi], \\
u(0, t)=u(\pi, t)=0, \quad t \in J,
\end{gathered}
$$

where $0<\alpha \leq 1,0<t_{1}<\cdots<t_{m}<a, c_{k}, k=1, \ldots, m$, are given constants and the function $b(t, s)$ is continuous on $\Delta$. Let us take the function $g(t, u(\cdot))=x \arctan u(x, \cdot)$, the multivalued map $f(t, s, \cdot)=b(t, s) e$, the nonlocal function given by $h(u(\cdot, t))=\sum_{k=1}^{m} c_{k} u\left(\cdot, t_{k}\right)$, the control functions $\mu_{1}(t)=\mu_{2}(t)=\xi(\cdot, t)$, where $\xi:[0, \pi] \times J \rightarrow[0, \pi]$ is continuous, and the delays $\sigma(t)=\delta(t)=\sin t$. Assume that $H=L^{2}[0, \pi]$ and define $A: H \rightarrow H$ by $A w=w^{\prime \prime}$ with domain

$$
D(A)=\left\{w \in H: w, w^{\prime} \text { are absolutely continuous, } w^{\prime \prime} \in H, w(0)=w(\pi)=0\right\}
$$

dense in the Hilbert space $H$. Then,

$$
A w=\sum_{n=1}^{\infty} n^{2}\left\langle w, w_{n}\right\rangle w_{n}, \quad w \in D(A),
$$

where $\langle\cdot, \cdot\rangle$ is the inner product in $L^{2}[0, \pi]$. It is well known that $A$ generates a strongly continuous semigroup $\{Q(t), t \geq 0\}$ on $H$, which is compact, analytic, and self-adjoint. Furthermore, $A$ has a 
discrete spectrum with eigenvalues $-n^{2}, n \in \mathbb{N}$, and the corresponding normalized eigenfunctions are given by $w_{n}(t)=\sqrt{\frac{2}{\pi}} \sin n x, 0 \leq x \leq \pi$, with $\left\{w_{n}: n \in \mathbb{N}\right\}$ an orthonormal basis of $H$ and

$$
Q(t) w=\sum_{n=1}^{\infty} e^{-n^{2} t}\left\langle w, w_{n}\right\rangle w_{n}
$$

for all $t \geq 0$ and $w \in H$. In particular, $Q(\cdot)$ is a uniformly stable semigroup and $\|Q(t)\|_{L^{2}[0, \pi]} \leq e^{-t}$. Also, for each $w \in H, A^{-\frac{1}{2}} w=\sum_{n=1}^{\infty} \frac{1}{n}\left\langle w, w_{n}\right\rangle w_{n}$ with $\left\|A^{-\frac{1}{2}}\right\|_{L^{2}[0, \pi]}=1$ and the operator $A^{\frac{1}{2}}$ is given on the space $D\left(A^{\frac{1}{2}}\right)=H_{\frac{1}{2}}:=\left\{w \in H: \sum_{n=1}^{\infty} n\left\langle w, w_{n}\right\rangle w_{n} \in H\right\}$ by $A^{\frac{1}{2}} w=\sum_{n=1}^{\infty} n\left\langle w, w_{n}\right\rangle w_{n}$. Now define the infinite-dimensional space $Y$ by

$$
Y:=\left\{z=\sum_{n=2}^{\infty} z_{n} w_{n}(x) \mid \sum_{n=2}^{\infty} z_{n}<\infty\right\} \subset L^{2}[0, \pi],
$$

where the norm in $Y$ is defined by $\|z\|=\sqrt{\sum_{n=2}^{\infty} z_{n}^{2}}$. The control is defined by a bounded linear operator $B=B_{1}=B_{2}: Y \rightarrow L^{2}[0, \pi]$ from the control Hilbert space $Y$ by

$$
(B \mu)(x)=2 \mu_{2} w_{1}(x)+\sum_{n=2}^{\infty} \mu_{n} w_{n}(x)=\xi(x, t), \quad \text { for } \quad z=\sum_{n=2}^{\infty} \mu_{n} w_{n} \in Y .
$$

Assume that there exists a function $v(x, \delta(t))=\omega(x, \sin t)$ such that

$$
\omega(x, \sin t)+\xi(x, t) \in \int_{0}^{t} b(t, s) \exp \xi(x, \sin s) d s .
$$

Thus, the integral equation (5) is satisfied. Therefore, problem (16)-(18) is an abstract formulation of the control system (11) -(2). Moreover, all the assumptions $\left(\mathrm{H}_{1}\right)-\left(\mathrm{H}_{4}\right)$ hold. Then, the associated linear system of (16)-(18) is not exactly controllable but, by Theorems 15 and 16, the control system (16)-(18) is approximately controllable on $J$. Note that Lemma 14 holds.

\section{Conclusion}

We have studied approximate controllability for a class of fractional delay dynamic inclusions. We introduced, for the first time in the literature, a nonlocal control condition by setting a control function that depends on the nonlocal condition, and other control that depends on the multivalued map that appears, as usual, in the right hand side of the inclusion. Sufficient conditions for approximate controllability are obtained. In particular, our conditions are formulated in such a way that approximate controllability of the nonlinear dynamical system is implied by the approximate controllability of its corresponding linear part. More precisely, the controllability problem is transformed into a fixed point problem for an appropriate nonlinear operator in a suitable function space. Using fractional calculations, multi-valued analysis, and Sadovskii's fixed point theorem, we guarantee the existence of a fixed point of this operator and study approximate controllability of the considered systems. Finally, an example is provided to illustrate the applicability of the new results.

In order to describe various real-world problems in physical and engineering sciences subject to abrupt changes at certain instants during the evolution process, impulsive fractional differential equations have become important in recent years as mathematical models of many phenomena in both physical and social sciences [43. In [11, Debbouche and Baleanu establish a controllability result for a class of fractional evolution nonlocal impulsive quasilinear delay integro-differential systems in a Banach space by using the theory of fractional calculus and fixed point techniques. Upon making some appropriate assumptions on system functions and by adapting the techniques 
and ideas established here with those of [11, one can prove approximate controllability of nonlocal control fractional delay dynamic inclusions with impulses.

Degenerate differential equations of integer order are often used to describe various processes in science and engineering [19]. As an open problem for further investigations, we mention the study of approximate controllability for degenerate fractional dynamic (stochastic) inclusions.

\section{Acknowledgments}

This work was supported by Portuguese funds through the Center for Research and Development in Mathematics and Applications (CIDMA), and The Portuguese Foundation for Science and Technology (FCT), within project PEst-OE/MAT/UI4106/2014. Debbouche was also supported by FCT within the post-doc project BPD/UA/CIDMA/2011 — PD2012-MTSC. The hospitality and the excellent working conditions at the University of Aveiro and CIDMA are gratefully acknowledged. The authors would like also to thank the reviewers for their valuable comments.

\section{References}

[1] N. Abada, M. Benchohra and H. Hammouche, Existence and controllability results for nondensely defined impulsive semilinear functional differential inclusions, J. Differential Equations 246 (2009), no. $10,3834-3863$.

[2] R. P. Agarwal, M. Benchohra and S. Hamani, A survey on existence results for boundary value problems of nonlinear fractional differential equations and inclusions, Acta Appl. Math. 109 (2010), no. 3, 973-1033.

[3] R. L. Bagley and P. J. Torvik, A theoretical basis for the application of fractional calculus to viscoelasticity, J. Rheology 27 (1983), 201-210.

[4] R. L. Bagley and P. J. Torvik, Fractional calculus in the transient analysis of viscoelastically damped structures, Amer. Inst. Aeronaut. Astronaut. 23 (1985), 918-925.

[5] D. Baleanu, K. Diethelm, E. Scalas and J. J. Trujillo, Fractional calculus, Series on Complexity, Nonlinearity and Chaos, 3, World Scientific Publishing Co. Pte. Ltd., Hackensack, NJ, 2012.

[6] A. E. Bashirov and N. I. Mahmudov, On concepts of controllability for deterministic and stochastic systems, SIAM J. Control Optim. 37 (1999), no. 6, 1808-1821.

[7] M. Benchohra, E. P. Gatsori and S. K. Ntouyas, Controllability results for semilinear evolution inclusions with nonlocal conditions, J. Optim. Theory Appl. 118 (2003), no. 3, 493-513.

[8] M. Benchohra and N. Hamidi, Fractional order differential inclusions via the topological transversality method, Cubo 13 (2011), no. 2, 139-149.

[9] T. S. Chow, Fractional dynamics of interfaces between soft-nanoparticles and rough substrates, Phys. Lett. A 342 (2005), 148-155.

[10] A. Debbouche, Fractional nonlocal impulsive quasilinear multi-delay integro-differential systems, Adv. Difference Equ. 2011 (2011), no. 5, 10 pp.

[11] A. Debbouche and D. Baleanu, Controllability of fractional evolution nonlocal impulsive quasilinear delay integro-differential systems, Comput. Math. Appl. 62 (2011), no. 3, 1442-1450.

[12] A. Debbouche and D. Baleanu, Exact null controllability for fractional nonlocal integrodifferential equations via implicit evolution system, J. Appl. Math. 2012 (2012), Art. ID 931975, 17 pp.

[13] A. Debbouche, D. Baleanu and R. P. Agarwal, Nonlocal nonlinear integrodifferential equations of fractional orders, Bound. Value Probl. 2012 (2012), no. 78, 10 pp.

[14] A. Debbouche and M. M. El-Borai, Weak almost periodic and optimal mild solutions of fractional evolution equations, Electron. J. Differential Equations 2009 (2009), no. 46, 8 pp.

[15] A. Debbouche and D. F. M. Torres, Approximate controllability of fractional nonlocal delay semilinear systems in Hilbert spaces, Internat. J. Control 86 (2013), no. 9, 1577-1585. arXiv:1304.0082

[16] K. Deimling, Multivalued differential equations, de Gruyter Series in Nonlinear Analysis and Applications, 1, de Gruyter, Berlin, 1992. 
[17] M. M. El-Borai, Some probability densities and fundamental solutions of fractional evolution equations, Chaos Solitons Fractals 14 (2002), no. 3, 433-440.

[18] A. M. A. El-Sayed, Fractional-order diffusion-wave equation, Internat. J. Theoret. Phys. 35 (1996), no. 2, 311-322.

[19] V. E. Fedorov and A. Debbouche, A class of degenerate fractional evolution systems in Banach spaces, Differ. Equ. 49 (2013), no. 12, 1569-1576.

[20] X. Fu, Approximate controllability for neutral impulsive differential inclusions with nonlocal conditions, J. Dyn. Control Syst. 17 (2011), no. 3, 359-386.

[21] J. H. He, Nonlinear oscillation with fractional derivative and its applications, in: International Conference on Vibrating Engineering '98, Dalian, China (1998), 288-291.

[22] R. Hilfer, Applications of fractional calculus in physics, World Sci. Publishing, River Edge, NJ, 2000.

[23] E. Hille and R. S. Phillips, Functional analysis and semi-groups, American Mathematical Society Colloquium Publications, vol. 31, Amer. Math. Soc., Providence, RI, 1957.

[24] M. Kamenskii, V. Obukhovskii and P. Zecca, Condensing multivalued maps and semilinear differential inclusions in Banach spaces, De Gruyter Series in Nonlinear Analysis and Applications, 7, de Gruyter, Berlin, 2001.

[25] A. A. Kilbas, H. M. Srivastava and J. J. Trujillo, Theory and applications of fractional differential equations, North-Holland Mathematics Studies, 204, Elsevier, Amsterdam, 2006.

[26] S. Kumar and N. Sukavanam, Approximate controllability of fractional order semilinear systems with bounded delay, J. Differential Equations 252 (2012), no. 11, 6163-6174.

[27] A. Lasota and Z. Opial, An application of the Kakutani-Ky Fan theorem in the theory of ordinary differential equations, Bull. Acad. Polon. Sci. Sér. Sci. Math. Astronom. Phys. 13 (1965), 781-786.

[28] F. Li, T.-J. Xiao and H.-K. Xu, On nonlinear neutral fractional integrodifferential inclusions with infinite delay, J. Appl. Math. 2012 (2012), Art. ID 916543, 19 pp.

[29] R. L. Magin, Fractional calculus in bioengineering, Critical Rev. Biomed. Eng. 32 (2004), 1-377.

[30] F. Mainardi, Fractional calculus: some basic problems in continuum and statistical mechanics, in Fractals and fractional calculus in continuum mechanics (Udine, 1996), 291-348, CISM Courses and Lectures, 378 Springer, Vienna, 1997.

[31] A. B. Malinowska and D. F. M. Torres, Introduction to the fractional calculus of variations, Imp. Coll. Press, London, 2012.

[32] K. S. Miller and B. Ross, An introduction to the fractional calculus and fractional differential equations, A Wiley-Interscience Publication, Wiley, New York, 1993.

[33] D. Mozyrska and D. F. M. Torres, Minimal modified energy control for fractional linear control systems with the Caputo derivative, Carpathian J. Math. 26 (2010), no. 2, 210-221. arXiv:1004.3113

[34] D. Mozyrska and D. F. M. Torres, Modified optimal energy and initial memory of fractional continuous-time linear systems, Signal Process., 91 (2011), no. 3, 379-385. arXiv:1007.3946

[35] S. K. Ntouyas and D. O'Regan, Existence results for semilinear neutral functional differential inclusions via analytic semigroups, Acta Appl. Math. 98 (2007), no. 3, 223-253.

[36] M. D. Ortigueira, On the initial conditions in continuous time fractional linear systems, Signal Process. 83 (2003), 2301-2309.

[37] N. S. Papageorgiou, On the theory of Banach space valued multifunctions. I. Integration and conditional expectation, J. Multivariate Anal. 17 (1985), no. 2, 185-206.

[38] A. Pazy, Semigroups of linear operators and applications to partial differential equations, Applied Mathematical Sciences, 44, Springer, New York, 1983.

[39] I. Podlubny, Fractional differential equations, Mathematics in Science and Engineering, 198, Academic Press, San Diego, CA, 1999.

[40] B. Radhakrishnan and K. Balachandran, Controllability of neutral evolution integrodifferential systems with state dependent delay, J. Optim. Theory Appl. 153 (2012), no. 1, 85-97.

[41] S. Rathinasamy and R. Yong, Approximate controllability of fractional differential equations with state-dependent delay, Results Math. 63 (2013), no. 3-4, 949-963. 
[42] J. Sabatier, O. P. Agrawal and J. A. T. Machado (Eds.), Advances in fractional calculus, Springer, Dordrecht, 2007.

[43] R. Sakthivel, R. Ganesh and S. M. Anthoni, Approximate controllability of fractional nonlinear differential inclusions, Appl. Math. Comput. 225 (2013), 708-717.

[44] R. Sakthivel, R. Ganesh, Y. Ren and S. M. Anthoni, Approximate controllability of nonlinear fractional dynamical systems, Commun. Nonlinear Sci. Numer. Simul. 18 (2013), no. 12, 3498-3508.

[45] R. Sakthivel, R. Ganesh and S. Suganya, Approximate controllability of fractional neutral stochastic system with infinite delay, Rep. Math. Phys. 70 (2012), no. 3, 291-311.

[46] R. Sakthivel, N. I. Mahmudov and Juan. J. Nieto, Controllability for a class of fractional-order neutral evolution control systems, Appl. Math. Comput. 218 (2012), no. 20, 10334-10340.

[47] R. Sakthivel, Y. Ren and N. I. Mahmudov, On the approximate controllability of semilinear fractional differential systems, Comput. Math. Appl. 62 (2011), no. 3, 1451-1459.

[48] R. Sakthivel, S. Suganya and S. M. Anthoni, Approximate controllability of fractional stochastic evolution equations, Comput. Math. Appl. 63 (2012), no. 3, 660-668.

[49] N. Sukavanam and S. Kumar, Approximate controllability of fractional order semilinear delay systems, J. Optim. Theory Appl. 151 (2011), no. 2, 373-384.

[50] R. Triggiani, A note on the lack of exact controllability for mild solutions in Banach spaces, SIAM J. Control Optimization 15 (1977), no. 3, 407-411.

[51] Y. Wang and D. Li, Morse Decompositions for Periodic General Dynamical Systems and Differential Inclusions, Set-Valued Var. Anal. 20 (2012), no. 4, 519-549.

[52] Z. Yan, Approximate controllability of partial neutral functional differential systems of fractional order with state-dependent delay, Internat. J. Control 85 (2012), no. 8, 1051-1062.

[53] Z. Yan, Approximate controllability of fractional neutral integro-differential inclusions with statedependent delay in Hilbert spaces, IMA J. Math. Control Inform. 30 (2013), no. 4, 443-462.

[54] S. D. Zaidman, Abstract differential equations, Research Notes in Mathematics, 36, Pitman, Boston, MA, 1979.

[55] Y. Zhou and F. Jiao, Existence of mild solutions for fractional neutral evolution equations, Comput. Math. Appl. 59 (2010), no. 3, 1063-1077. 